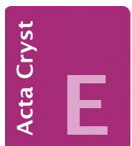

CRYSTALLOGRAPHIC COMMUNICATIONS

ISSN 2056-9890

Received 8 April 2016

Accepted 21 April 2016

Edited by D.-J. Xu, Zhejiang University (Yuquan Campus), China

Keywords: crystal structure; canagliflozin; hydrogen bonding.

CCDC reference: 1475516

Supporting information: this article has supporting information at journals.iucr.org/e

\section{Crystal structure of canagliflozin hemihydrate}

\author{
Kai-Hang Liu, ${ }^{\mathrm{a}}$ Jian-Ming Gu, ${ }^{\mathrm{b}}$ Xiu-Rong $\mathrm{Hu}^{\mathrm{a} *}$ and Gu-Ping Tang ${ }^{\mathrm{a}}$
}

${ }^{a}$ Chemistry Department, Zhejiang University, Hangzhou, Zhejiang 310028, People's Republic of China, and ${ }^{\mathbf{b} C e n t e r}$ of Analysis and Measurement, Zhejiang University, Hangzhou, Zhejiang 310028, People's Republic of China.

*Correspondence e-mail: huxiurong@zju.edu.cn

There are two canagliflozin molecules $(A$ and $B)$ and one water molecule in the asymmetric unit of the title compound, $\mathrm{C}_{24} \mathrm{H}_{25} \mathrm{FO}_{5} \mathrm{~S} \cdot 0.5 \mathrm{H}_{2} \mathrm{O}$ [systematic name: $(2 S, 3 R, 4 R, 5 S, 6 R)-2-(3-\{[5-(4-f l u o r o p h e n y l) t h i o p h e n-2-y l] m e t h y l\}-4-m e t h y l$ phenyl)-6-(hydroxymethyl)-3,4,5,6-tetrahydro- $2 H$-pyran-3,4,5-triol hemihydrate]. The dihedral angles between the methylbenzene and thiophene rings are $115.7(4)$ and $111.7(4)^{\circ}$, while the dihedral angles between the fluorobenzene and thiophene rings are $24.2(6)$ and $20.5(9)^{\circ}$ in molecules $A$ and $B$, respectively. The hydropyran ring exhibits a chair conformation in both canagliflozin molecules. In the crystal, the canagliflozin molecules and lattice water molecules are connected via $\mathrm{O}-\mathrm{H} \cdots \mathrm{O}$ hydrogen bonds into a threedimensional supramolecular architecture.

\section{Chemical context}

Canagliflozin is a member of a new class of anti-diabetic drugs which are used to improve glycemic control of diabetics (Cefalu et al., 2013). The crystalline forms of canagliflozin have been reported (Mitsubishi et al., 2013; Ahmed et al., 2013; Chen et al., 2013), we report here the single-crystal structure of the title compound.<smiles>CCCCCOC(O)C1OCC(O)C(O)C1O</smiles>

\section{Structural commentary}

The title compound crystallizes with two independent canagliflozin molecules and one water molecule in the asymmetric unit (Fig. 1). The water molecule links the two canagliflozin molecules $(A$ and $B)$ via two $\mathrm{O}-\mathrm{H} \cdots \mathrm{O}$ hydrogen bonds (Table 1).

The conformations of the two canagliflozin molecules are somewhat different with regard to the orientation of the central benzene ring $(\mathrm{C} 12-\mathrm{C} 17)$ with respect to the thiophene ring, as indicated by torsion angles $\mathrm{C} 9 A-\mathrm{C} 10 A-\mathrm{C} 11 A-$ $\mathrm{C} 12 A=113.3(6)^{\circ}$ in molecule $A$ and $\mathrm{C} 9 B-\mathrm{C} 10 B-\mathrm{C} 11 B-$ $\mathrm{C} 12 B=108.0(6)^{\circ}$ in molecule $B$. The conformational difference is also shown by the angle $\mathrm{C} 10-\mathrm{C} 11-\mathrm{C} 12$, which is $115.7(4)^{\circ}$ in molecule $A$ and $111.7(4)^{\circ}$ in molecule $B$. The terminal aromatic rings $(\mathrm{C} 1-\mathrm{C} 6)$ are inclined to the thiophene rings, forming dihedral angles of $24.2(6)$ and $20.5(9)^{\circ}$ in molecules $A$ and $B$, respectively. The tetrahydropyran rings 


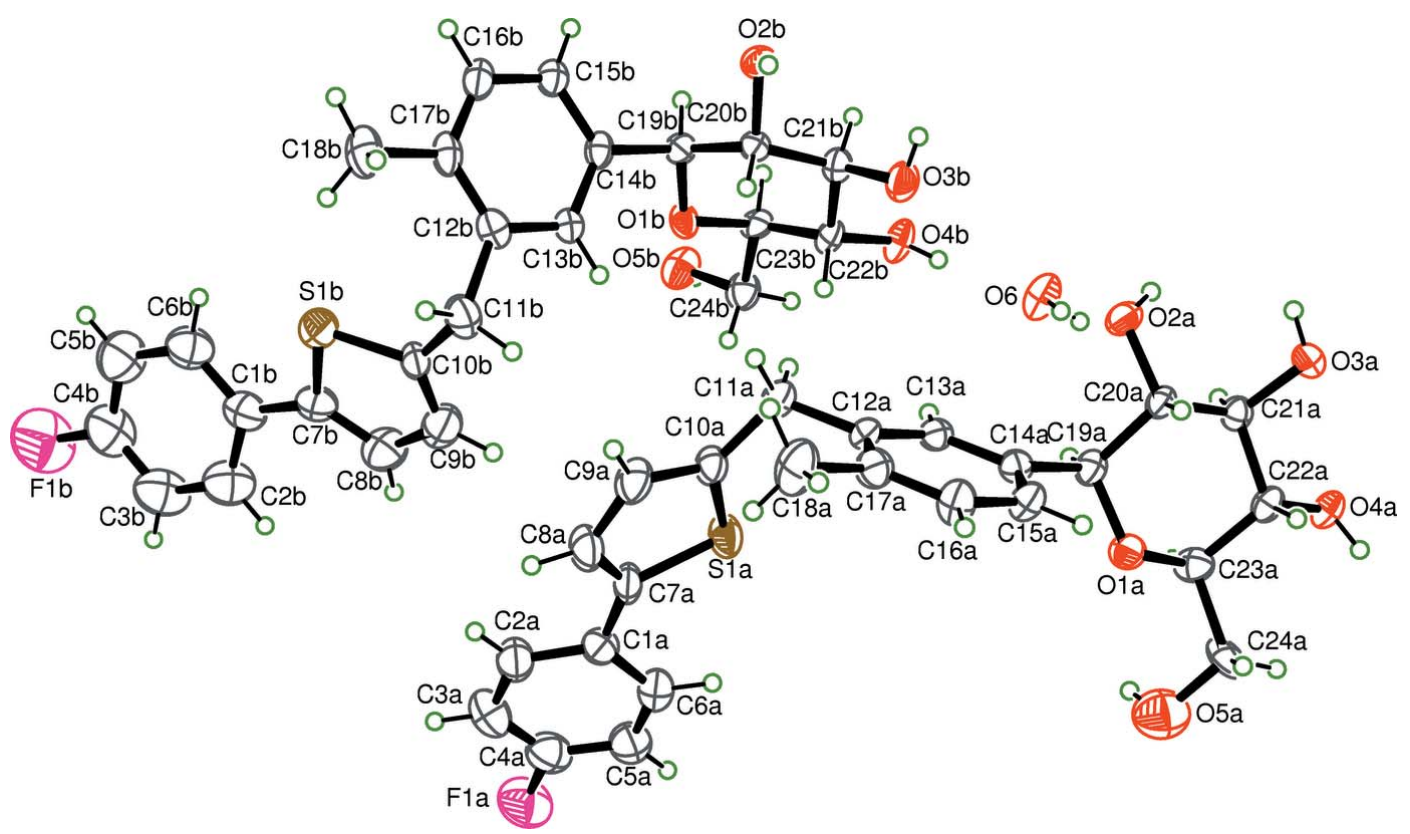

Figure 1

The molecular structure of the title compound, (I), showing the atom-labeling scheme and displacement ellipsoids at the $40 \%$ probability level. $\mathrm{H}$ atoms are shown as small circles of arbitrary radii.

exhibit a distorted chair conformation in both molecules $A$ and $B$.

\section{Supramolecular features}

In the crystal, $\mathrm{O} 3 B-\mathrm{H} 3 B 1 \cdots \mathrm{O} 4 B^{\mathrm{i}}, \mathrm{O} 2 B-\mathrm{H} 2 B 1 \cdots \mathrm{O} 4 A^{\mathrm{iii}}$, and $\mathrm{O} 5 B-\mathrm{H} 5 B 1 \cdots \mathrm{O} 3 B^{\text {iv }}$ [symmetry code: (i) $x-\frac{1}{2},-y+\frac{3}{2},-z+1$; (iii) $x, y+1, z$; (iv) $x+1, y, z]$ link canagliflozin molecules, generating a ring of graph-set motif $R_{3}^{3}(9)$. The presence of the water molecules results in the formation of zigzag chains mediated by alternating $\mathrm{O} 4 B-\mathrm{H} 4 B \cdots \mathrm{O} 6$, O6 $-\mathrm{H} 61 \cdots \mathrm{O} 2 A$ and $\mathrm{O} 4 A-\mathrm{H} 4 A \cdots \mathrm{O} 5 B^{\mathrm{ii}}$ [symmetry code: (ii) $x-1, y-1, z$ ] hydrogen bonds propagating along the $a$ axis; the chains are stacked along the $c$ axis by further hydrogen-bonding interactions, $\mathrm{O} 3 A-\mathrm{H} 3 A 1 \cdots \mathrm{O} 2 B^{\mathrm{i}}$ and $\mathrm{O} 2 A--\mathrm{H} 2 A 1 \cdots \mathrm{O} 2 B^{\mathrm{i}}$ (Fig. 2).

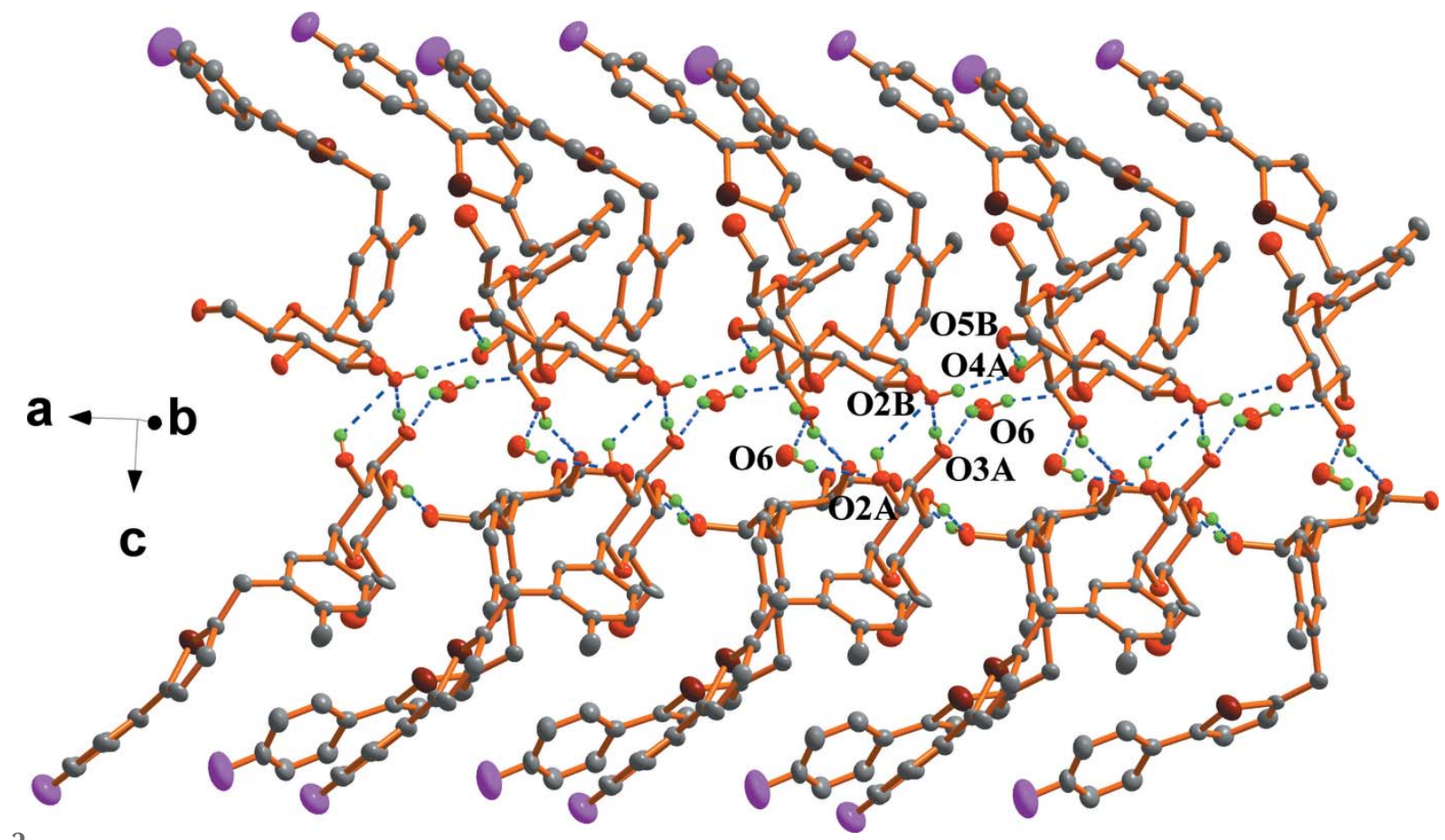

Figure 2

Part of the crystal packing of the title compound, showing the extensive intermolecular hydrogen-bonding interactions (dashed lines). $\mathrm{H}$ atoms not involved in hydrogen bonding have been omitted for clarity. 
Table 1

Hydrogen-bond geometry $\left(\AA,^{\circ}\right)$.

\begin{tabular}{lllll}
\hline$D-\mathrm{H} \cdots A$ & $D-\mathrm{H}$ & $\mathrm{H} \cdots A$ & $D \cdots A$ & $D-\mathrm{H} \cdots A$ \\
\hline $\mathrm{O} 2 A-\mathrm{H} 2 A 1 \cdots \mathrm{O} 2 B^{\mathrm{i}}$ & 0.82 & 2.42 & $2.841(4)$ & 113 \\
$\mathrm{O} 3 A-\mathrm{H} 3 A 1 \cdots \mathrm{O} 2 B^{\mathrm{i}}$ & 0.82 & 2.17 & $2.951(4)$ & 158 \\
$\mathrm{O} 4 A-\mathrm{H} 4 A \cdots \mathrm{O} 5 B^{\mathrm{ii}}$ & 0.82 & 1.98 & $2.756(5)$ & 157 \\
$\mathrm{O} 2 B-\mathrm{H} 2 B 1 \cdots \mathrm{O} 4 A^{\mathrm{iii}}$ & 0.82 & 1.85 & $2.672(4)$ & 179 \\
$\mathrm{O} 3 B-\mathrm{H} 3 B 1 \cdots \mathrm{O} 4 B^{\mathrm{i}}$ & 0.82 & 1.99 & $2.797(4)$ & 168 \\
$\mathrm{O} 4 B-\mathrm{H} 4 B \cdots \mathrm{O} 6$ & 0.82 & 1.93 & $2.749(5)$ & 172 \\
$\mathrm{O} 5 B-\mathrm{H} 5 B 1 \cdots \mathrm{O} 3 B^{\mathrm{iv}}$ & 0.82 & 2.31 & $3.015(5)$ & 144 \\
$\mathrm{O} 6-\mathrm{H} 61 \cdots \mathrm{O} 2 A$ & 0.82 & 2.23 & $3.031(5)$ & 166 \\
$\mathrm{O} 6-\mathrm{H} 62 \cdots \mathrm{O} 3 A^{\mathrm{v}}$ & 0.83 & 2.30 & $3.058(5)$ & 153 \\
\hline
\end{tabular}

Symmetry codes: (i) $x-\frac{1}{2},-y+\frac{3}{2},-z+1$; (ii) $x-1, y-1, z$; (iii) $x, y+1, z$; (iv) $x+1, y, z ;(\mathrm{v}) x+\frac{1}{2},-y+\frac{1}{2},-z+1$.

\section{Synthesis and crystallization}

The crude product was supplied by Zhejiang Huadong Pharmaceutical Co., Ltd. It was recrystallized from methanol solution, giving colorless crystals suitable for X-ray diffraction.

\section{Refinement}

Crystal data, data collection and structure refinement details are summarized in Table 2. All $\mathrm{H}$ atoms were placed in calculated positions with $\mathrm{C}-\mathrm{H}=0.93-0.98 \AA$ and $\mathrm{O}-\mathrm{H}=$ $0.82 \AA$ and included in the refinement using a riding model, with $U_{\text {iso }}(\mathrm{H})=1.2 U_{\text {eq }}$ or $1.5 U_{\text {eq }}$ (carrier atom).

\section{Acknowledgements}

This project was supported by the Analysis and Measurement Foundation of Zhejiang Province, China (grant No. 2014 C37055).

\section{References}

Ahmed, F. A., Maureen, C., Steven, M., Lorraine, S., Kenneth, M. W., Fan, Z., Sumihiro, N., Mitsuya, H. \& Yuichi, K. (2013). US Patent 2009/0233874 A1.

Brandenburg, K. \& Putz, H. (2005). DIAMOND. Crystal Impact GbR, Bonn, Germany.
Table 2

Experimental details.

\begin{tabular}{ll}
\hline Crystal data & \\
Chemical formula & $2 \mathrm{C}_{24} \mathrm{H}_{25} \mathrm{FO}_{5} \mathrm{~S} \cdot \mathrm{H}_{2} \mathrm{O}$ \\
$M_{\mathrm{r}}$ & 907.02 \\
Crystal system, space group & Orthorhombic, $P 2_{1} 2_{1} 2_{1}$ \\
Temperature $(\mathrm{K})$ & 296 \\
$a, b, c(\AA)$ & $8.4259(4), 11.4264(7), 45.706(2)$ \\
$V\left(\AA^{3}\right)$ & $4400.4(4)$ \\
$Z$ & 4 \\
Radiation type & Mo $K \alpha$ \\
$\mu\left(\mathrm{mm}^{-1}\right)$ & 0.19 \\
Crystal size (mm) & $0.48 \times 0.28 \times 0.26$ \\
& \\
Data collection & Rigaku R-AXIS RAPID \\
Diffractometer & Multi-scan $(A B S C O R ;$ Higashi, \\
Absorption correction & $1995)$ \\
& $0.914,0.952$ \\
$T_{\text {min }}, T_{\text {max }}$ & $43211,9958,5079$ \\
No. of measured, independent and & \\
$\quad$ observed $[I>2 \sigma(I)]$ reflections & 0.145 \\
$R_{\text {int }}$ & 0.649 \\
(sin $\theta / \lambda)_{\text {max }}\left(\AA^{-1}\right)$ & \\
Refinement & \\
$R\left[F^{2}>2 \sigma\left(F^{2}\right)\right], w R\left(F^{2}\right), S$ & $0.080,0.147,1.00$ \\
No. of reflections & 9958 \\
No. of parameters & 575 \\
H-atom treatment & $\mathrm{H}-$ atom parameters constrained \\
$\Delta \rho_{\max }, \Delta \rho_{\min }\left(\mathrm{e} \AA^{-3}\right)$ & $0.38,-0.29$ \\
Absolute structure & Flack $(1983), 3246$ Friedel pairs \\
Absolute structure parameter & $0.13(11)$ \\
\hline &
\end{tabular}

Computer programs: PROCESS-AUTO (Rigaku, 2006), CrystalStructure (Rigaku, 2007), SHELXS97 and SHELXL97 (Sheldrick, 2008), ORTEP-3 for Windows and WinGX (Farrugia, 2012) and DIAMOND (Brandenburg \& Putz, 2005).

Cefalu, W. T., Leiter, L. A., Yoon, K. H., Arias, P., Niskanen, L., Xie, J., Balis, D. A., Canovatchel, W. \& Meininger, G. (2013). Lancet, 382, 941-950.

Chen, M.-H., Zhang, Y.-F., Zhao, Y. \& Zhang, X.-Y. (2013). Chin. Patent CN103588762A.

Farrugia, L. J. (2012). J. Appl. Cryst. 45, 849-854.

Flack, H. D. (1983). Acta Cryst. A39, 876-881.

Higashi, T. (1995). ABSCORM. Rigaku Corporation, Tokyo, Japan.

Mitsubishi, T., Nomura, S. \& Kawanishi, A. (2013). World Patent WO2008069327A1.

Rigaku (2006). PROCESS-AUTO. Rigaku Corporation, Tokyo, Japan.

Rigaku. (2007). CrystalStructure. Rigaku Americas, The Woodlands, Texas, USA.

Sheldrick, G. M. (2008). Acta Cryst. A64, 112-122. 


\section{supporting information}

Acta Cryst. (2016). E72, 734-736 [doi:10.1107/S2056989016006769]

\section{Crystal structure of canagliflozin hemihydrate}

\section{Kai-Hang Liu, Jian-Ming Gu, Xiu-Rong Hu and Gu-Ping Tang}

Computing details

Data collection: PROCESS-AUTO (Rigaku, 2006); cell refinement: PROCESS-AUTO (Rigaku, 2006); data reduction: CrystalStructure (Rigaku, 2007); program(s) used to solve structure: SHELXS97 (Sheldrick, 2008); program(s) used to refine structure: SHELXL97 (Sheldrick, 2008); molecular graphics: ORTEP-3 for Windows (Farrugia, 2012) and DIAMOND (Brandenburg \& Putz, 2005); software used to prepare material for publication: WinGX (Farrugia, 2012).

(2S,3R,4R,5S,6R)-2-(3-\{[5-(4-Fluorophenyl)thiophen-2-yl] methyl\}-4-methylphenyl)-6-(hydroxymethyl)-3,4,5,6tetrahydro- $2 H$-pyran-3,4,5-triol hemihydrate

Crystal data

$2 \mathrm{C}_{24} \mathrm{H}_{25} \mathrm{FO}_{5} \mathrm{~S} \cdot \mathrm{H}_{2} \mathrm{O}$

$M_{r}=907.02$

Orthorhombic, $P 2_{1} 2_{1} 2_{1}$

Hall symbol: P 2ac 2ab

$a=8.4259(4) \AA$

$b=11.4264(7) \AA$

$c=45.706(2) \AA$

$V=4400.4(4) \AA^{3}$

$Z=4$

\section{Data collection}

\section{Rigaku R-AXIS RAPID}

diffractometer

Radiation source: rotating anode

Graphite monochromator

Detector resolution: 10.00 pixels $\mathrm{mm}^{-1}$

$\omega$ scans

Absorption correction: multi-scan

(ABSCOR; Higashi, 1995)

$T_{\min }=0.914, T_{\max }=0.952$

Refinement

Refinement on $F^{2}$

Least-squares matrix: full

$R\left[F^{2}>2 \sigma\left(F^{2}\right)\right]=0.080$

$w R\left(F^{2}\right)=0.147$

$S=1.00$

9958 reflections

575 parameters

0 restraints

Primary atom site location: structure-invariant direct methods
$F(000)=1912$

$D_{\mathrm{x}}=1.369 \mathrm{Mg} \mathrm{m}^{-3}$

Mo $K \alpha$ radiation, $\lambda=0.71073 \AA$

Cell parameters from 23292 reflections

$\theta=3.0-27.4^{\circ}$

$\mu=0.19 \mathrm{~mm}^{-1}$

$T=296 \mathrm{~K}$

Needle, colorless

$0.48 \times 0.28 \times 0.26 \mathrm{~mm}$

43211 measured reflections

9958 independent reflections

5079 reflections with $I>2 \sigma(I)$

$R_{\text {int }}=0.145$

$\theta_{\max }=27.5^{\circ}, \theta_{\min }=3.0^{\circ}$

$h=-10 \rightarrow 10$

$k=-14 \rightarrow 14$

$l=-59 \rightarrow 59$

Secondary atom site location: difference Fourier map

Hydrogen site location: inferred from neighbouring sites

$\mathrm{H}$-atom parameters constrained

$w=1 /\left[\sigma^{2}\left(F_{\mathrm{o}}^{2}\right)+(0.0408 P)^{2}+2.8647 P\right]$

where $P=\left(F_{\mathrm{o}}{ }^{2}+2 F_{\mathrm{c}}{ }^{2}\right) / 3$

$(\Delta / \sigma)_{\max }<0.001$

$\Delta \rho_{\max }=0.38 \mathrm{e} \AA^{-3}$

$\Delta \rho_{\min }=-0.29$ e $\AA^{-3}$ 
Absolute structure: Flack (1983), 3246 Friedel pairs

\section{Special details}

Geometry. All esds (except the esd in the dihedral angle between two 1.s. planes) are estimated using the full covariance matrix. The cell esds are taken into account individually in the estimation of esds in distances, angles and torsion angles; correlations between esds in cell parameters are only used when they are defined by crystal symmetry. An approximate (isotropic) treatment of cell esds is used for estimating esds involving l.s. planes.

Refinement. Refinement of $\mathrm{F}^{2}$ against ALL reflections. The weighted R-factor $\mathrm{wR}$ and goodness of fit $\mathrm{S}$ are based on $\mathrm{F}^{2}$, conventional R-factors $R$ are based on $F$, with $F$ set to zero for negative $F^{2}$. The threshold expression of $F^{2}>2 \operatorname{sigma}\left(F^{2}\right)$ is used only for calculating R-factors(gt) etc. and is not relevant to the choice of reflections for refinement. R-factors based on $\mathrm{F}^{2}$ are statistically about twice as large as those based on F, and R- factors based on ALL data will be even larger.

Fractional atomic coordinates and isotropic or equivalent isotropic displacement parameters $\left(\AA^{2}\right)$

\begin{tabular}{|c|c|c|c|c|}
\hline & $x$ & $y$ & $z$ & $U_{\text {iso }} * / U_{\text {eq }}$ \\
\hline $\mathrm{C} 1 \mathrm{~A}$ & $0.8230(6)$ & $0.7018(5)$ & $0.29229(10)$ & $0.0474(13)$ \\
\hline $\mathrm{C} 2 \mathrm{~A}$ & $0.9098(7)$ & $0.7759(5)$ & $0.27372(11)$ & $0.0595(16)$ \\
\hline $\mathrm{H} 2 \mathrm{~A}$ & 0.8808 & 0.8542 & 0.2721 & $0.071^{*}$ \\
\hline $\mathrm{C} 3 \mathrm{~A}$ & $1.0373(7)$ & $0.7347(6)$ & $0.25788(12)$ & $0.0692(17)$ \\
\hline $\mathrm{H} 3 \mathrm{~A}$ & 1.0956 & 0.7843 & 0.2458 & $0.083 *$ \\
\hline $\mathrm{C} 4 \mathrm{~A}$ & $1.0755(7)$ & $0.6195(7)$ & $0.26042(13)$ & $0.0676(18)$ \\
\hline C5A & $0.9969(7)$ & $0.5431(5)$ & $0.27796(12)$ & $0.0652(16)$ \\
\hline $\mathrm{H} 5 \mathrm{~A}$ & 1.0277 & 0.4651 & 0.2793 & $0.078 *$ \\
\hline C6A & $0.8699(7)$ & $0.5855(5)$ & $0.29361(11)$ & $0.0601(15)$ \\
\hline H6A & 0.8134 & 0.5343 & 0.3055 & $0.072 *$ \\
\hline C7A & $0.6928(6)$ & $0.7483(5)$ & $0.31027(9)$ & $0.0451(12)$ \\
\hline C8A & $0.6052(6)$ & $0.8464(5)$ & $0.30734(11)$ & $0.0554(15)$ \\
\hline H8A & 0.6133 & 0.8964 & 0.2914 & $0.066^{*}$ \\
\hline C9A & $0.4994(6)$ & $0.8657(5)$ & $0.33098(12)$ & $0.0557(15)$ \\
\hline H9A & 0.4306 & 0.9293 & 0.3318 & $0.067 *$ \\
\hline $\mathrm{C} 10 \mathrm{~A}$ & $0.5076(6)$ & $0.7834(4)$ & $0.35224(10)$ & $0.0421(12)$ \\
\hline $\mathrm{C} 11 \mathrm{~A}$ & $0.4200(6)$ & $0.7753(4)$ & $0.38050(10)$ & 0.0477 (13) \\
\hline H11A & 0.3621 & 0.8478 & 0.3834 & $0.057 *$ \\
\hline H11B & 0.4969 & 0.7688 & 0.3962 & $0.057 *$ \\
\hline C12A & $0.3033(5)$ & $0.6737(4)$ & $0.38309(9)$ & $0.0369(11)$ \\
\hline C13A & $0.3383(5)$ & $0.5781(4)$ & $0.40050(9)$ & 0.0343 (11) \\
\hline $\mathrm{H} 13 \mathrm{~A}$ & 0.4332 & 0.5779 & 0.4109 & $0.041^{*}$ \\
\hline C14A & $0.2385(5)$ & $0.4834(4)$ & $0.40311(9)$ & $0.0369(11)$ \\
\hline $\mathrm{C} 15 \mathrm{~A}$ & $0.0979(5)$ & $0.4846(4)$ & $0.38708(10)$ & $0.0404(12)$ \\
\hline H15A & 0.0283 & 0.4216 & 0.3883 & $0.049^{*}$ \\
\hline C16A & $0.0611(6)$ & $0.5791(5)$ & $0.36942(10)$ & $0.0482(13)$ \\
\hline H16A & -0.0332 & 0.5784 & 0.3588 & $0.058 *$ \\
\hline C17A & $0.1607(6)$ & $0.6745(5)$ & $0.36711(10)$ & $0.0463(12)$ \\
\hline C18A & $0.1135(7)$ & $0.7778(5)$ & $0.34833(12)$ & $0.0690(17)$ \\
\hline $\mathrm{H} 18 \mathrm{~A}$ & 0.0041 & 0.7703 & 0.3428 & $0.104 *$ \\
\hline H18B & 0.1787 & 0.7798 & 0.3311 & $0.104^{*}$ \\
\hline $\mathrm{H} 18 \mathrm{C}$ & 0.1278 & 0.8489 & 0.3592 & $0.104 *$ \\
\hline C19A & $0.2741(5)$ & $0.3786(4)$ & $0.42179(9)$ & $0.0357(11)$ \\
\hline
\end{tabular}




\begin{tabular}{|c|c|c|c|c|}
\hline H19A & 0.3865 & 0.3797 & 0.4273 & $0.043^{*}$ \\
\hline $\mathrm{C} 20 \mathrm{~A}$ & $0.1737(5)$ & $0.3719(4)$ & $0.44938(9)$ & $0.0326(11)$ \\
\hline $\mathrm{H} 20 \mathrm{~A}$ & 0.0615 & 0.3757 & 0.4438 & $0.039^{*}$ \\
\hline C21A & $0.2017(5)$ & $0.2584(4)$ & $0.46571(8)$ & $0.0339(10)$ \\
\hline $\mathrm{H} 21 \mathrm{~A}$ & 0.3096 & 0.2591 & 0.4737 & $0.041^{*}$ \\
\hline $\mathrm{C} 22 \mathrm{~A}$ & $0.1845(5)$ & $0.1543(4)$ & $0.44575(9)$ & $0.0340(11)$ \\
\hline $\mathrm{H} 22 \mathrm{~A}$ & 0.0726 & 0.1443 & 0.4405 & $0.041^{*}$ \\
\hline $\mathrm{C} 23 \mathrm{~A}$ & $0.2830(6)$ & 0.1693 (4) & $0.41808(9)$ & $0.0424(12)$ \\
\hline $\mathrm{H} 23 \mathrm{~A}$ & 0.3968 & 0.1644 & 0.4223 & $0.051^{*}$ \\
\hline $\mathrm{C} 24 \mathrm{~A}$ & $0.2307(7)$ & $0.0731(5)$ & $0.39562(10)$ & $0.0588(13)$ \\
\hline $\mathrm{H} 24 \mathrm{~A}$ & 0.2386 & -0.0039 & 0.4045 & $0.071^{*}$ \\
\hline H24B & 0.1215 & 0.0858 & 0.3897 & $0.071^{*}$ \\
\hline C1B & $1.2588(6)$ & 1.2409 (5) & 0.30205 (11) & $0.0562(14)$ \\
\hline $\mathrm{C} 2 \mathrm{~B}$ & $1.3509(7)$ & $1.1863(6)$ & $0.28096(13)$ & 0.0768 (19) \\
\hline $\mathrm{H} 2 \mathrm{~B}$ & 1.3240 & 1.1110 & 0.2751 & $0.092 *$ \\
\hline $\mathrm{C} 3 \mathrm{~B}$ & $1.4806(7)$ & $1.2395(8)$ & $0.26844(14)$ & $0.085(2)$ \\
\hline H3B & 1.5430 & 1.2007 & 0.2548 & $0.102^{*}$ \\
\hline C4B & $1.5136(8)$ & $1.3496(8)$ & $0.27672(16)$ & $0.085(2)$ \\
\hline C5B & $1.4341(8)$ & $1.4082(7)$ & $0.29795(16)$ & $0.086(2)$ \\
\hline H5B & 1.4656 & 1.4824 & 0.3039 & $0.103^{*}$ \\
\hline C6B & $1.3035(7)$ & $1.3528(6)$ & $0.31044(13)$ & $0.0697(17)$ \\
\hline H6B & 1.2451 & 1.3917 & 0.3247 & $0.084 *$ \\
\hline C7B & $1.1148(6)$ & $1.1843(5)$ & $0.31376(10)$ & $0.0499(13)$ \\
\hline $\mathrm{C} 8 \mathrm{~B}$ & $1.0747(7)$ & $1.0717(6)$ & $0.31352(12)$ & $0.0637(16)$ \\
\hline H8B & 1.1397 & 1.0133 & 0.3060 & $0.076^{*}$ \\
\hline C9B & $0.9242(7)$ & $1.0495(5)$ & $0.32585(12)$ & $0.0627(16)$ \\
\hline H9B & 0.8806 & 0.9750 & 0.3274 & $0.075^{*}$ \\
\hline C10B & 0.8499 (6) & $1.1468(5)$ & $0.33522(9)$ & $0.0441(13)$ \\
\hline C11B & $0.6910(6)$ & $1.1544(5)$ & $0.35001(10)$ & $0.0532(14)$ \\
\hline $\mathrm{H} 11 \mathrm{C}$ & 0.6363 & 1.0801 & 0.3480 & $0.064^{*}$ \\
\hline H11D & 0.6274 & 1.2139 & 0.3404 & $0.064^{*}$ \\
\hline C12B & $0.7072(5)$ & $1.1844(5)$ & $0.38244(9)$ & $0.0389(12)$ \\
\hline C13B & $0.7355(5)$ & 1.0932 (4) & $0.40164(9)$ & $0.0355(11)$ \\
\hline H13B & 0.7394 & 1.0170 & 0.3945 & $0.043^{*}$ \\
\hline C14B & $0.7583(5)$ & $1.1132(4)$ & $0.43165(9)$ & 0.0334 (11) \\
\hline C15B & $0.7535(5)$ & $1.2272(4)$ & $0.44152(10)$ & $0.0373(11)$ \\
\hline H15B & 0.7706 & 1.2431 & 0.4612 & $0.045^{*}$ \\
\hline C16B & $0.7237(5)$ & $1.3172(4)$ & $0.42246(10)$ & $0.0437(12)$ \\
\hline H16B & 0.7195 & 1.3933 & 0.4296 & $0.052 *$ \\
\hline $\mathrm{C} 17 \mathrm{~B}$ & $0.6997(5)$ & $1.2985(4)$ & $0.39290(11)$ & $0.0411(12)$ \\
\hline C18B & $0.6624(6)$ & $1.4007(5)$ & $0.37319(11)$ & $0.0586(15)$ \\
\hline H18D & 0.6825 & 1.4725 & 0.3835 & $0.088^{*}$ \\
\hline H18E & 0.5528 & 1.3977 & 0.3675 & $0.088^{*}$ \\
\hline $\mathrm{H} 18 \mathrm{~F}$ & 0.7281 & 1.3971 & 0.3561 & $0.088^{*}$ \\
\hline C19B & $0.7807(5)$ & $1.0121(4)$ & $0.45231(9)$ & $0.0330(10)$ \\
\hline H19B & 0.8318 & 1.0399 & 0.4703 & $0.040^{*}$ \\
\hline $\mathrm{C} 20 \mathrm{~B}$ & $0.6217(5)$ & $0.9556(4)$ & $0.45993(9)$ & $0.0302(10)$ \\
\hline H20B & 0.5595 & 0.9465 & 0.4420 & $0.036^{*}$ \\
\hline
\end{tabular}




$\begin{array}{lllll}\text { C21B } & 0.6378(5) & 0.8365(4) & 0.47464(9) & 0.0353(11) \\ \text { H21B } & 0.6707 & 0.8489 & 0.4950 & 0.042^{*} \\ \text { C22B } & 0.7590(5) & 0.7601(4) & 0.46008(9) & 0.0341(10) \\ \text { H22B } & 0.7200 & 0.7347 & 0.4409 & 0.041^{*} \\ \text { C23B } & 0.9139(5) & 0.8280(4) & 0.45649(9) & 0.0335(11) \\ \text { H23B } & 0.9519 & 0.8539 & 0.4757 & 0.040^{*} \\ \text { C24B } & 1.0424(5) & 0.7610(4) & 0.44114(10) & 0.0466(12) \\ \text { H24C } & 1.0593 & 0.6864 & 0.4508 & 0.056^{*} \\ \text { H24D } & 1.0119 & 0.7461 & 0.4210 & 0.056^{*} \\ \text { F1A } & 1.2033(4) & 0.5781(4) & 0.24514(8) & 0.0935(12) \\ \text { F1B } & 1.6394(5) & 1.4058(5) & 0.26352(10) & 0.1351(18) \\ \text { O1A } & 0.2438(4) & 0.2775(3) & 0.40429(6) & 0.0421(8) \\ \text { O2A } & 0.2095(4) & 0.4714(3) & 0.46711(6) & 0.0440(8) \\ \text { H2A1 } & 0.2400 & 0.4495 & 0.4832 & 0.066^{*} \\ \text { O3A } & 0.0913(4) & 0.2435(3) & 0.48929(6) & 0.0441(8) \\ \text { H3A1 } & 0.0983 & 0.2991 & 0.5006 & 0.066^{*} \\ \text { O4A } & 0.2376(4) & 0.0535(3) & 0.46192(7) & 0.0406(8) \\ \text { H4A } & 0.1973 & -0.0057 & 0.4550 & 0.061^{*} \\ \text { O5A } & 0.3311(6) & 0.0803(4) & 0.37124(10) & 0.0913(14) \\ \text { H5A1 } & 0.3019 & 0.1340 & 0.3606 & 0.137^{*} \\ \text { O1B } & 0.8812(3) & 0.9285(3) & 0.43833(6) & 0.0370(7) \\ \text { O2B } & 0.5393(3) & 1.0343(3) & 0.47925(6) & 0.0371(8) \\ \text { H2B1 } & 0.4465 & 1.0401 & 0.4741 & 0.056^{*} \\ \text { O3B } & 0.4879(4) & 0.7782(3) & 0.47478(7) & 0.0472(8) \\ \text { H3B1 } & 0.4316 & 0.8062 & 0.4876 & 0.071^{*} \\ \text { O4B } & 0.7951(4) & 0.6600(3) & 0.47784(7) & 0.0463(8) \\ \text { H4B } & 0.7223 & 0.6126 & 0.4768 & 0.069^{*} \\ \text { O5B } & 1.1853(4) & 0.8292(3) & 0.44195(8) & 0.0559(9) \\ \text { H5B1 } & 1.2601 & 0.7872 & 0.4466 & 0.084^{*} \\ \text { O6 } & 0.5619(4) & 0.4920(3) & 0.47940(8) & 0.0602(10) \\ \text { H61 } & 0.4711 & 0.4818 & 0.4733 & 0.090^{*} \\ \text { H62 } & 0.5714 & 0.4204 & 0.4819 & 0.090^{*} \\ \text { S1A } & 0.64414(16) & 0.67821(12) & 0.34263(3) & 0.0523(4) \\ \text { S1B } & 0.96635(17) & 1.26679(13) & 0.32902(3) & 0.0598(4) \\ & & & & \end{array}$

Atomic displacement parameters $\left(\AA^{2}\right)$

\begin{tabular}{lllllll}
\hline & $U^{11}$ & $U^{22}$ & $U^{33}$ & $U^{12}$ & $U^{13}$ & $U^{23}$ \\
\hline C1A & $0.055(3)$ & $0.047(4)$ & $0.041(3)$ & $-0.002(3)$ & $-0.004(3)$ & $0.002(2)$ \\
C2A & $0.067(4)$ & $0.057(4)$ & $0.054(3)$ & $-0.010(3)$ & $-0.002(3)$ & $0.010(3)$ \\
C3A & $0.063(4)$ & $0.085(5)$ & $0.060(4)$ & $0.000(4)$ & $0.014(3)$ & $0.011(4)$ \\
C4A & $0.060(4)$ & $0.083(5)$ & $0.060(4)$ & $-0.005(4)$ & $0.005(3)$ & $-0.011(4)$ \\
C5A & $0.077(4)$ & $0.057(4)$ & $0.062(4)$ & $0.005(4)$ & $0.010(4)$ & $-0.002(3)$ \\
C6A & $0.071(4)$ & $0.049(4)$ & $0.060(3)$ & $-0.009(3)$ & $0.009(3)$ & $0.006(3)$ \\
C7A & $0.052(3)$ & $0.039(3)$ & $0.044(3)$ & $-0.014(3)$ & $0.000(2)$ & $0.013(3)$ \\
C8A & $0.066(4)$ & $0.045(4)$ & $0.055(3)$ & $0.006(3)$ & $0.010(3)$ & $0.017(3)$ \\
C9A & $0.062(4)$ & $0.035(3)$ & $0.070(4)$ & $0.007(3)$ & $0.003(3)$ & $0.009(3)$ \\
C10A & $0.047(3)$ & $0.032(3)$ & $0.048(3)$ & $0.005(2)$ & $0.006(2)$ & $0.008(2)$
\end{tabular}




\begin{tabular}{|c|c|c|c|c|c|c|}
\hline C11A & $0.061(3)$ & $0.030(3)$ & $0.052(3)$ & $-0.002(3)$ & 0.005 & $0.000(2)$ \\
\hline $\mathrm{C} 12 \mathrm{~A}$ & 0.047 (3) & $0.025(3)$ & $0.038(2)$ & $-0.004(2)$ & $0.008(2)$ & $-0.005(2)$ \\
\hline $\mathrm{C} 13 \mathrm{~A}$ & 0.033 & $0.035(3)$ & $0.035(2)$ & $-0.001(2)$ & $0.001(2)$ & $-0.004(2)$ \\
\hline $\mathrm{C} 14 \mathrm{~A}$ & $0.042(3)$ & $0.033(3)$ & $0.036(2)$ & $0.002(2)$ & $0.004(2)$ & $0.000(2)$ \\
\hline $\mathrm{C} 15 \mathrm{~A}$ & $0.042(3)$ & $0.031(3)$ & $0.048(3)$ & $-0.003(2)$ & $-0.009(2)$ & $0.003(2)$ \\
\hline $\mathrm{C} 16 \mathrm{~A}$ & $0.045(3)$ & $0.047(3)$ & $0.053(3)$ & $-0.004(3)$ & $-0.013(3)$ & 0.010 \\
\hline $\mathrm{C} 17 \mathrm{~A}$ & $0.055(3)$ & $0.042(3)$ & $0.042(3)$ & $0.002(3)$ & $-0.005(3)$ & $0.005(2)$ \\
\hline C18A & $0.074(4)$ & $0.050(4)$ & $0.083(4)$ & $-0.001(3)$ & $-0.020(3)$ & $0.021(3)$ \\
\hline C19A & $0.034(3)$ & $0.029(3)$ & $0.044(3)$ & $0.000(2)$ & $0.002(2)$ & $0.002(2)$ \\
\hline $\mathrm{C} 20 \mathrm{~A}$ & 0.037 (3) & 0.023 & $0.038(2)$ & $-0.002(2)$ & $0.000(2)$ & $-0.001(2)$ \\
\hline $\mathrm{C} 21 \mathrm{~A}$ & $0.033(2)$ & 0.033 & $0.036(2)$ & $-0.003(2)$ & $-0.004(2)$ & $0.003(2)$ \\
\hline $\mathrm{C} 22 \mathrm{~A}$ & $0.033(2)$ & $0.024(3)$ & $0.045(3)$ & $0.002(2)$ & -0.005 (2) & $0.002(2)$ \\
\hline $\mathrm{C} 23 \mathrm{~A}$ & 0.049 (3) & $0.037(3)$ & $0.042(3)$ & $0.010(3)$ & $-0.003(2)$ & $-0.002(2)$ \\
\hline $\mathrm{C} 24 \mathrm{~A}$ & 0.086 & 0.044 & $0.046(3)$ & $0.026(3)$ & $0.038(3)$ & 0.008 (3) \\
\hline $\mathrm{C} 1 \mathrm{~B}$ & $0.058(3)$ & $0.063(4)$ & $0.047(3)$ & $0.017(3)$ & -0.003 & 0.002 \\
\hline $\mathrm{C} 2 \mathrm{~B}$ & $0.062(4)$ & $0.089(5)$ & $0.079(4)$ & $0.006(4)$ & $0.001(4)$ & $-0.013(4)$ \\
\hline $\mathrm{C} 3 \mathrm{~B}$ & $0.056(4)$ & $0.116(7)$ & $0.082(5)$ & $0.004(5)$ & $0.012(4)$ & $-0.016(5)$ \\
\hline C4B & $0.051(4)$ & $0.116(7)$ & $0.088(5)$ & -0.020 & 0.008 (4) & $0.000(5)$ \\
\hline C5B & $0.070(5)$ & $0.090(6)$ & $0.098(5)$ & $-0.013(4)$ & $0.001(4)$ & $-0.001(5)$ \\
\hline C6B & 0.064 (4) & $0.078(5)$ & 0.068 (4) & $0.006(4)$ & $0.009(3)$ & -0.007 (3) \\
\hline C7B & $0.048(3)$ & $0.056(4)$ & $0.046(3)$ & $0.008(3)$ & $0.003(3)$ & $-0.006(3)$ \\
\hline $\mathrm{C} 8 \mathrm{~B}$ & $0.062(4)$ & $0.056(4)$ & $0.073(4)$ & $0.016(3)$ & $0.002(3)$ & $-0.003(3)$ \\
\hline C9B & $0.075(4)$ & $0.049(4)$ & $0.064(4)$ & $-0.006(3)$ & $-0.012(3)$ & 0.004 (3) \\
\hline $\mathrm{C} 10 \mathrm{~B}$ & 0.058 & $0.043(3)$ & $0.032(3)$ & $-0.014(3)$ & $0.000(2)$ & 0.009 (2) \\
\hline C11B & $0.057(3)$ & $0.058(4)$ & 0.045 & $-0.006(3)$ & $-0.002(3)$ & 0.011 \\
\hline C12B & $0.031(2)$ & $0.051(3)$ & $0.035(2)$ & $-0.003(2)$ & $-0.004(2)$ & $0.007(2)$ \\
\hline C13B & $0.040(3)$ & 0.033 & $0.034(2)$ & $-0.002(2)$ & $0.002(2)$ & $0.005(2)$ \\
\hline C14B & $0.028(2)$ & $0.029(3)$ & $0.043(3)$ & $0.003(2)$ & $0.003(2)$ & $0.009(2)$ \\
\hline C15B & $0.036(2)$ & $0.034(3)$ & $0.042(2)$ & $0.002(2)$ & $0.004(2)$ & $0.006(2)$ \\
\hline $\mathrm{C} 16 \mathrm{~B}$ & $0.040(3)$ & $0.032(3)$ & $0.059(3)$ & $0.002(2)$ & $0.013(3)$ & $0.008(3)$ \\
\hline C17B & $0.033(3)$ & $0.038(3)$ & $0.052(3)$ & $0.006(2)$ & $0.007(2)$ & $0.018(2)$ \\
\hline $\mathrm{C} 18 \mathrm{~B}$ & $0.055(3)$ & $0.050(4)$ & $0.070(4)$ & $0.014(3)$ & 0.005 & $0.026(3)$ \\
\hline C19B & $0.037(3)$ & $0.028(3)$ & $0.034(2)$ & $0.006(2)$ & $0.001(2)$ & $0.002(2)$ \\
\hline $\mathrm{C} 20 \mathrm{~B}$ & $0.030(2)$ & $0.031(3)$ & $0.029(2)$ & $0.001(2)$ & $0.000(2)$ & $-0.0001(19)$ \\
\hline $\mathrm{C} 21 \mathrm{~B}$ & $0.034(2)$ & $0.034(3)$ & $0.038(2)$ & $-0.005(2)$ & $0.000(2)$ & $0.004(2)$ \\
\hline $\mathrm{C} 22 \mathrm{~B}$ & $0.038(3)$ & $0.023(3)$ & $0.041(2)$ & $-0.002(2)$ & $-0.007(2)$ & $0.005(2)$ \\
\hline $\mathrm{C} 23 \mathrm{~B}$ & $0.040(3)$ & $0.022(2)$ & $0.038(2)$ & $0.011(2)$ & $0.000(2)$ & $-0.001(2)$ \\
\hline $\mathrm{C} 24 \mathrm{~B}$ & $0.045(3)$ & $0.038(3)$ & $0.056(3)$ & $0.004(3)$ & $0.003(2)$ & $0.006(3)$ \\
\hline F1A & $0.074(2)$ & $0.115(3)$ & $0.091(2)$ & $0.010(2)$ & $0.028(2)$ & $-0.011(2)$ \\
\hline F1B & $0.076(3)$ & $0.183(5)$ & $0.146(4)$ & $-0.037(3)$ & $0.029(3)$ & $-0.007(4)$ \\
\hline O1A & $0.056(2)$ & $0.0316(19)$ & $0.0392(16)$ & $0.0070(17)$ & $0.0008(15)$ & $0.0012(15)$ \\
\hline $\mathrm{O} 2 \mathrm{~A}$ & $0.061(2)$ & $0.031(2)$ & $0.0395(17)$ & $-0.0058(17)$ & $-0.0058(17)$ & $-0.0042(15)$ \\
\hline $\mathrm{O} 3 \mathrm{~A}$ & $0.057(2)$ & $0.039(2)$ & $0.0357(17)$ & $-0.0041(18)$ & $0.0126(15)$ & $0.0011(16)$ \\
\hline $\mathrm{O} 4 \mathrm{~A}$ & $0.045(2)$ & $0.0237(18)$ & $0.0527(19)$ & $-0.0026(16)$ & $-0.0045(16)$ & $0.0017(15)$ \\
\hline $\mathrm{O} 5 \mathrm{~A}$ & $0.101(4)$ & $0.085(4)$ & $0.088(3)$ & $0.020(3)$ & $0.006(3)$ & $-0.006(3)$ \\
\hline O1B & $0.0411(17)$ & $0.0312(19)$ & $0.0388(16)$ & $0.0069(15)$ & $0.0085(15)$ & $0.0103(15)$ \\
\hline $\mathrm{O} 2 \mathrm{~B}$ & $0.0341(16)$ & $0.037(2)$ & $0.0405(17)$ & $0.0026(15)$ & $0.0010(15)$ & $-0.0046(15)$ \\
\hline $\mathrm{O} 3 \mathrm{~B}$ & 0.0437 (19) & $0.040(2)$ & $0.058(2)$ & $-0.0110(17)$ & $0.0070(16)$ & $0.0015(16)$ \\
\hline
\end{tabular}




\begin{tabular}{lllllll} 
O4B & $0.047(2)$ & $0.0264(19)$ & $0.066(2)$ & $-0.0040(16)$ & $-0.0110(18)$ & $0.0141(17)$ \\
O5B & $0.042(2)$ & $0.042(2)$ & $0.084(3)$ & $0.0068(18)$ & $0.009(2)$ & $-0.002(2)$ \\
O6 & $0.062(2)$ & $0.041(2)$ & $0.079(3)$ & $-0.0114(19)$ & $-0.011(2)$ & $0.007(2)$ \\
S1A & $0.0602(8)$ & $0.0419(8)$ & $0.0549(8)$ & $0.0073(7)$ & $0.0109(7)$ & $0.0161(7)$ \\
S1B & $0.0673(9)$ & $0.0499(10)$ & $0.0621(8)$ & $-0.0040(8)$ & $0.0172(7)$ & $-0.0030(7)$ \\
\hline
\end{tabular}

Geometric parameters $\left(\AA,{ }^{\circ}\right)$

\begin{tabular}{|c|c|c|c|}
\hline $\mathrm{C} 1 \mathrm{~A}-\mathrm{C} 6 \mathrm{~A}$ & $1.387(7)$ & $\mathrm{C} 3 \mathrm{~B}-\mathrm{C} 4 \mathrm{~B}$ & $1.344(9)$ \\
\hline $\mathrm{C} 1 \mathrm{~A}-\mathrm{C} 2 \mathrm{~A}$ & $1.405(7)$ & $\mathrm{C} 3 \mathrm{~B}-\mathrm{H} 3 \mathrm{~B}$ & 0.9300 \\
\hline $\mathrm{C} 1 \mathrm{~A}-\mathrm{C} 7 \mathrm{~A}$ & $1.470(7)$ & $\mathrm{C} 4 \mathrm{~B}-\mathrm{C} 5 \mathrm{~B}$ & $1.356(9)$ \\
\hline $\mathrm{C} 2 \mathrm{~A}-\mathrm{C} 3 \mathrm{~A}$ & $1.378(8)$ & $\mathrm{C} 4 \mathrm{~B}-\mathrm{F} 1 \mathrm{~B}$ & $1.378(7)$ \\
\hline $\mathrm{C} 2 \mathrm{~A}-\mathrm{H} 2 \mathrm{~A}$ & 0.9300 & $\mathrm{C} 5 \mathrm{~B}-\mathrm{C} 6 \mathrm{~B}$ & $1.391(8)$ \\
\hline $\mathrm{C} 3 \mathrm{~A}-\mathrm{C} 4 \mathrm{~A}$ & $1.360(8)$ & $\mathrm{C} 5 \mathrm{~B}-\mathrm{H} 5 \mathrm{~B}$ & 0.9300 \\
\hline $\mathrm{C} 3 \mathrm{~A}-\mathrm{H} 3 \mathrm{~A}$ & 0.9300 & $\mathrm{C} 6 \mathrm{~B}-\mathrm{H} 6 \mathrm{~B}$ & 0.9300 \\
\hline $\mathrm{C} 4 \mathrm{~A}-\mathrm{C} 5 \mathrm{~A}$ & $1.358(8)$ & $\mathrm{C} 7 \mathrm{~B}-\mathrm{C} 8 \mathrm{~B}$ & $1.330(8)$ \\
\hline $\mathrm{C} 4 \mathrm{~A}-\mathrm{F} 1 \mathrm{~A}$ & $1.368(7)$ & $\mathrm{C} 7 \mathrm{~B}-\mathrm{S} 1 \mathrm{~B}$ & $1.715(5)$ \\
\hline $\mathrm{C} 5 \mathrm{~A}-\mathrm{C} 6 \mathrm{~A}$ & $1.376(8)$ & $\mathrm{C} 8 \mathrm{~B}-\mathrm{C} 9 \mathrm{~B}$ & $1.411(8)$ \\
\hline $\mathrm{C} 5 \mathrm{~A}-\mathrm{H} 5 \mathrm{~A}$ & 0.9300 & $\mathrm{C} 8 \mathrm{~B}-\mathrm{H} 8 \mathrm{~B}$ & 0.9300 \\
\hline $\mathrm{C} 6 \mathrm{~A}-\mathrm{H} 6 \mathrm{~A}$ & 0.9300 & $\mathrm{C} 9 \mathrm{~B}-\mathrm{C} 10 \mathrm{~B}$ & $1.345(7)$ \\
\hline $\mathrm{C} 7 \mathrm{~A}-\mathrm{C} 8 \mathrm{~A}$ & $1.348(7)$ & $\mathrm{C} 9 \mathrm{~B}-\mathrm{H} 9 \mathrm{~B}$ & 0.9300 \\
\hline $\mathrm{C} 7 \mathrm{~A}-\mathrm{S} 1 \mathrm{~A}$ & $1.731(4)$ & $\mathrm{C} 10 \mathrm{~B}-\mathrm{C} 11 \mathrm{~B}$ & $1.502(7)$ \\
\hline $\mathrm{C} 8 \mathrm{~A}-\mathrm{C} 9 \mathrm{~A}$ & $1.418(7)$ & $\mathrm{C} 10 \mathrm{~B}-\mathrm{S} 1 \mathrm{~B}$ & $1.710(5)$ \\
\hline $\mathrm{C} 8 \mathrm{~A}-\mathrm{H} 8 \mathrm{~A}$ & 0.9300 & $\mathrm{C} 11 \mathrm{~B}-\mathrm{C} 12 \mathrm{~B}$ & $1.527(6)$ \\
\hline $\mathrm{C} 9 \mathrm{~A}-\mathrm{C} 10 \mathrm{~A}$ & $1.354(6)$ & $\mathrm{C} 11 \mathrm{~B}-\mathrm{H} 11 \mathrm{C}$ & 0.9700 \\
\hline C9A-H9A & 0.9300 & $\mathrm{C} 11 \mathrm{~B}-\mathrm{H} 11 \mathrm{D}$ & 0.9700 \\
\hline $\mathrm{C} 10 \mathrm{~A}-\mathrm{C} 11 \mathrm{~A}$ & $1.491(6)$ & $\mathrm{C} 12 \mathrm{~B}-\mathrm{C} 13 \mathrm{~B}$ & $1.383(6)$ \\
\hline $\mathrm{C} 10 \mathrm{~A}-\mathrm{S} 1 \mathrm{~A}$ & $1.721(5)$ & $\mathrm{C} 12 \mathrm{~B}-\mathrm{C} 17 \mathrm{~B}$ & $1.390(6)$ \\
\hline $\mathrm{C} 11 \mathrm{~A}-\mathrm{C} 12 \mathrm{~A}$ & $1.526(6)$ & $\mathrm{C} 13 \mathrm{~B}-\mathrm{C} 14 \mathrm{~B}$ & $1.404(6)$ \\
\hline $\mathrm{C} 11 \mathrm{~A}-\mathrm{H} 11 \mathrm{~A}$ & 0.9700 & $\mathrm{C} 13 \mathrm{~B}-\mathrm{H} 13 \mathrm{~B}$ & 0.9300 \\
\hline $\mathrm{C} 11 \mathrm{~A}-\mathrm{H} 11 \mathrm{~B}$ & 0.9700 & $\mathrm{C} 14 \mathrm{~B}-\mathrm{C} 15 \mathrm{~B}$ & $1.379(6)$ \\
\hline $\mathrm{C} 12 \mathrm{~A}-\mathrm{C} 13 \mathrm{~A}$ & $1.383(6)$ & $\mathrm{C} 14 \mathrm{~B}-\mathrm{C} 19 \mathrm{~B}$ & $1.504(6)$ \\
\hline $\mathrm{C} 12 \mathrm{~A}-\mathrm{C} 17 \mathrm{~A}$ & $1.407(6)$ & $\mathrm{C} 15 \mathrm{~B}-\mathrm{C} 16 \mathrm{~B}$ & $1.371(6)$ \\
\hline $\mathrm{C} 13 \mathrm{~A}-\mathrm{C} 14 \mathrm{~A}$ & $1.375(6)$ & $\mathrm{C} 15 \mathrm{~B}-\mathrm{H} 15 \mathrm{~B}$ & 0.9300 \\
\hline $\mathrm{C} 13 \mathrm{~A}-\mathrm{H} 13 \mathrm{~A}$ & 0.9300 & $\mathrm{C} 16 \mathrm{~B}-\mathrm{C} 17 \mathrm{~B}$ & $1.383(6)$ \\
\hline $\mathrm{C} 14 \mathrm{~A}-\mathrm{C} 15 \mathrm{~A}$ & $1.394(6)$ & $\mathrm{C} 16 \mathrm{~B}-\mathrm{H} 16 \mathrm{~B}$ & 0.9300 \\
\hline $\mathrm{C} 14 \mathrm{~A}-\mathrm{C} 19 \mathrm{~A}$ & $1.501(6)$ & $\mathrm{C} 17 \mathrm{~B}-\mathrm{C} 18 \mathrm{~B}$ & $1.508(6)$ \\
\hline $\mathrm{C} 15 \mathrm{~A}-\mathrm{C} 16 \mathrm{~A}$ & $1.383(6)$ & $\mathrm{C} 18 \mathrm{~B}-\mathrm{H} 18 \mathrm{D}$ & 0.9600 \\
\hline $\mathrm{C} 15 \mathrm{~A}-\mathrm{H} 15 \mathrm{~A}$ & 0.9300 & $\mathrm{C} 18 \mathrm{~B}-\mathrm{H} 18 \mathrm{E}$ & 0.9600 \\
\hline $\mathrm{C} 16 \mathrm{~A}-\mathrm{C} 17 \mathrm{~A}$ & $1.379(7)$ & $\mathrm{C} 18 \mathrm{~B}-\mathrm{H} 18 \mathrm{~F}$ & 0.9600 \\
\hline $\mathrm{C} 16 \mathrm{~A}-\mathrm{H} 16 \mathrm{~A}$ & 0.9300 & $\mathrm{C} 19 \mathrm{~B}-\mathrm{O} 1 \mathrm{~B}$ & $1.427(5)$ \\
\hline $\mathrm{C} 17 \mathrm{~A}-\mathrm{C} 18 \mathrm{~A}$ & $1.513(7)$ & $\mathrm{C} 19 \mathrm{~B}-\mathrm{C} 20 \mathrm{~B}$ & $1.528(6)$ \\
\hline $\mathrm{C} 18 \mathrm{~A}-\mathrm{H} 18 \mathrm{~A}$ & 0.9600 & C19B-H19B & 0.9800 \\
\hline $\mathrm{C} 18 \mathrm{~A}-\mathrm{H} 18 \mathrm{~B}$ & 0.9600 & $\mathrm{C} 20 \mathrm{~B}-\mathrm{O} 2 \mathrm{~B}$ & $1.439(5)$ \\
\hline $\mathrm{C} 18 \mathrm{~A}-\mathrm{H} 18 \mathrm{C}$ & 0.9600 & $\mathrm{C} 20 \mathrm{~B}-\mathrm{C} 21 \mathrm{~B}$ & $1.524(6)$ \\
\hline $\mathrm{C} 19 \mathrm{~A}-\mathrm{O} 1 \mathrm{~A}$ & $1.428(5)$ & $\mathrm{C} 20 \mathrm{~B}-\mathrm{H} 20 \mathrm{~B}$ & 0.9800 \\
\hline $\mathrm{C} 19 \mathrm{~A}-\mathrm{C} 20 \mathrm{~A}$ & $1.521(6)$ & $\mathrm{C} 21 \mathrm{~B}-\mathrm{O} 3 \mathrm{~B}$ & $1.428(5)$ \\
\hline C19A-H19A & 0.9800 & $\mathrm{C} 21 \mathrm{~B}-\mathrm{C} 22 \mathrm{~B}$ & $1.499(6)$ \\
\hline
\end{tabular}




\begin{tabular}{|c|c|c|c|}
\hline $\mathrm{C} 20 \mathrm{~A}-\mathrm{O} 2 \mathrm{~A}$ & $1.428(5)$ & $\mathrm{C} 21 \mathrm{~B}-\mathrm{H} 21 \mathrm{~B}$ & 0.9800 \\
\hline $\mathrm{C} 20 \mathrm{~A}-\mathrm{C} 21 \mathrm{~A}$ & $1.515(6)$ & $\mathrm{C} 22 \mathrm{~B}-\mathrm{O} 4 \mathrm{~B}$ & $1.436(5)$ \\
\hline $\mathrm{C} 20 \mathrm{~A}-\mathrm{H} 20 \mathrm{~A}$ & 0.9800 & $\mathrm{C} 22 \mathrm{~B}-\mathrm{C} 23 \mathrm{~B}$ & $1.526(6)$ \\
\hline $\mathrm{C} 21 \mathrm{~A}-\mathrm{O} 3 \mathrm{~A}$ & $1.434(5)$ & $\mathrm{C} 22 \mathrm{~B}-\mathrm{H} 22 \mathrm{~B}$ & 0.9800 \\
\hline $\mathrm{C} 21 \mathrm{~A}-\mathrm{C} 22 \mathrm{~A}$ & $1.506(6)$ & $\mathrm{C} 23 \mathrm{~B}-\mathrm{O} 1 \mathrm{~B}$ & $1.444(5)$ \\
\hline $\mathrm{C} 21 \mathrm{~A}-\mathrm{H} 21 \mathrm{~A}$ & 0.9800 & $\mathrm{C} 23 \mathrm{~B}-\mathrm{C} 24 \mathrm{~B}$ & $1.500(6)$ \\
\hline $\mathrm{C} 22 \mathrm{~A}-\mathrm{O} 4 \mathrm{~A}$ & $1.439(5)$ & $\mathrm{C} 23 \mathrm{~B}-\mathrm{H} 23 \mathrm{~B}$ & 0.9800 \\
\hline $\mathrm{C} 22 \mathrm{~A}-\mathrm{C} 23 \mathrm{~A}$ & $1.522(6)$ & $\mathrm{C} 24 \mathrm{~B}-\mathrm{O} 5 \mathrm{~B}$ & $1.435(5)$ \\
\hline $\mathrm{C} 22 \mathrm{~A}-\mathrm{H} 22 \mathrm{~A}$ & 0.9800 & $\mathrm{C} 24 \mathrm{~B}-\mathrm{H} 24 \mathrm{C}$ & 0.9700 \\
\hline $\mathrm{C} 23 \mathrm{~A}-\mathrm{O} 1 \mathrm{~A}$ & $1.427(5)$ & $\mathrm{C} 24 \mathrm{~B}-\mathrm{H} 24 \mathrm{D}$ & 0.9700 \\
\hline $\mathrm{C} 23 \mathrm{~A}-\mathrm{C} 24 \mathrm{~A}$ & $1.567(7)$ & $\mathrm{O} 2 \mathrm{~A}-\mathrm{H} 2 \mathrm{~A} 1$ & 0.8200 \\
\hline $\mathrm{C} 23 \mathrm{~A}-\mathrm{H} 23 \mathrm{~A}$ & 0.9800 & $\mathrm{O} 3 \mathrm{~A}-\mathrm{H} 3 \mathrm{~A} 1$ & 0.8200 \\
\hline $\mathrm{C} 24 \mathrm{~A}-\mathrm{O} 5 \mathrm{~A}$ & $1.401(6)$ & $\mathrm{O} 4 \mathrm{~A}-\mathrm{H} 4 \mathrm{~A}$ & 0.8200 \\
\hline $\mathrm{C} 24 \mathrm{~A}-\mathrm{H} 24 \mathrm{~A}$ & 0.9700 & $\mathrm{O} 5 \mathrm{~A}-\mathrm{H} 5 \mathrm{~A} 1$ & 0.8200 \\
\hline $\mathrm{C} 24 \mathrm{~A}-\mathrm{H} 24 \mathrm{~B}$ & 0.9700 & $\mathrm{O} 2 \mathrm{~B}-\mathrm{H} 2 \mathrm{~B} 1$ & 0.8200 \\
\hline $\mathrm{C} 1 \mathrm{~B}-\mathrm{C} 2 \mathrm{~B}$ & $1.386(7)$ & $\mathrm{O} 3 \mathrm{~B}-\mathrm{H} 3 \mathrm{~B} 1$ & 0.8200 \\
\hline $\mathrm{C} 1 \mathrm{~B}-\mathrm{C} 6 \mathrm{~B}$ & $1.387(8)$ & $\mathrm{O} 4 \mathrm{~B}-\mathrm{H} 4 \mathrm{~B}$ & 0.8200 \\
\hline $\mathrm{C} 1 \mathrm{~B}-\mathrm{C} 7 \mathrm{~B}$ & $1.476(7)$ & $\mathrm{O} 5 \mathrm{~B}-\mathrm{H} 5 \mathrm{~B} 1$ & 0.8200 \\
\hline $\mathrm{C} 2 \mathrm{~B}-\mathrm{C} 3 \mathrm{~B}$ & $1.375(8)$ & O6-H61 & 0.8228 \\
\hline $\mathrm{C} 2 \mathrm{~B}-\mathrm{H} 2 \mathrm{~B}$ & 0.9300 & $\mathrm{O} 6-\mathrm{H} 62$ & 0.8292 \\
\hline $\mathrm{C} 6 \mathrm{~A}-\mathrm{C} 1 \mathrm{~A}-\mathrm{C} 2 \mathrm{~A}$ & $117.1(5)$ & $\mathrm{C} 4 \mathrm{~B}-\mathrm{C} 3 \mathrm{~B}-\mathrm{H} 3 \mathrm{~B}$ & 121.3 \\
\hline $\mathrm{C} 6 \mathrm{~A}-\mathrm{C} 1 \mathrm{~A}-\mathrm{C} 7 \mathrm{~A}$ & $122.3(5)$ & $\mathrm{C} 2 \mathrm{~B}-\mathrm{C} 3 \mathrm{~B}-\mathrm{H} 3 \mathrm{~B}$ & 121.3 \\
\hline $\mathrm{C} 2 \mathrm{~A}-\mathrm{C} 1 \mathrm{~A}-\mathrm{C} 7 \mathrm{~A}$ & $120.5(5)$ & $\mathrm{C} 3 \mathrm{~B}-\mathrm{C} 4 \mathrm{~B}-\mathrm{C} 5 \mathrm{~B}$ & $124.1(7)$ \\
\hline $\mathrm{C} 3 \mathrm{~A}-\mathrm{C} 2 \mathrm{~A}-\mathrm{C} 1 \mathrm{~A}$ & $121.1(6)$ & $\mathrm{C} 3 \mathrm{~B}-\mathrm{C} 4 \mathrm{~B}-\mathrm{F} 1 \mathrm{~B}$ & $118.2(7)$ \\
\hline $\mathrm{C} 3 \mathrm{~A}-\mathrm{C} 2 \mathrm{~A}-\mathrm{H} 2 \mathrm{~A}$ & 119.4 & $\mathrm{C} 5 \mathrm{~B}-\mathrm{C} 4 \mathrm{~B}-\mathrm{F} 1 \mathrm{~B}$ & $117.7(8)$ \\
\hline $\mathrm{C} 1 \mathrm{~A}-\mathrm{C} 2 \mathrm{~A}-\mathrm{H} 2 \mathrm{~A}$ & 119.4 & $\mathrm{C} 4 \mathrm{~B}-\mathrm{C} 5 \mathrm{~B}-\mathrm{C} 6 \mathrm{~B}$ & $117.4(7)$ \\
\hline $\mathrm{C} 4 \mathrm{~A}-\mathrm{C} 3 \mathrm{~A}-\mathrm{C} 2 \mathrm{~A}$ & $118.0(6)$ & $\mathrm{C} 4 \mathrm{~B}-\mathrm{C} 5 \mathrm{~B}-\mathrm{H} 5 \mathrm{~B}$ & 121.3 \\
\hline $\mathrm{C} 4 \mathrm{~A}-\mathrm{C} 3 \mathrm{~A}-\mathrm{H} 3 \mathrm{~A}$ & 121.0 & $\mathrm{C} 6 \mathrm{~B}-\mathrm{C} 5 \mathrm{~B}-\mathrm{H} 5 \mathrm{~B}$ & 121.3 \\
\hline $\mathrm{C} 2 \mathrm{~A}-\mathrm{C} 3 \mathrm{~A}-\mathrm{H} 3 \mathrm{~A}$ & 121.0 & $\mathrm{C} 1 \mathrm{~B}-\mathrm{C} 6 \mathrm{~B}-\mathrm{C} 5 \mathrm{~B}$ & $121.3(6)$ \\
\hline $\mathrm{C} 5 \mathrm{~A}-\mathrm{C} 4 \mathrm{~A}-\mathrm{C} 3 \mathrm{~A}$ & $123.9(6)$ & $\mathrm{C} 1 \mathrm{~B}-\mathrm{C} 6 \mathrm{~B}-\mathrm{H} 6 \mathrm{~B}$ & 119.3 \\
\hline $\mathrm{C} 5 \mathrm{~A}-\mathrm{C} 4 \mathrm{~A}-\mathrm{F} 1 \mathrm{~A}$ & $117.5(6)$ & $\mathrm{C} 5 \mathrm{~B}-\mathrm{C} 6 \mathrm{~B}-\mathrm{H} 6 \mathrm{~B}$ & 119.3 \\
\hline $\mathrm{C} 3 \mathrm{~A}-\mathrm{C} 4 \mathrm{~A}-\mathrm{F} 1 \mathrm{~A}$ & $118.5(6)$ & $\mathrm{C} 8 \mathrm{~B}-\mathrm{C} 7 \mathrm{~B}-\mathrm{C} 1 \mathrm{~B}$ & $129.0(5)$ \\
\hline $\mathrm{C} 4 \mathrm{~A}-\mathrm{C} 5 \mathrm{~A}-\mathrm{C} 6 \mathrm{~A}$ & $117.4(6)$ & $\mathrm{C} 8 \mathrm{~B}-\mathrm{C} 7 \mathrm{~B}-\mathrm{S} 1 \mathrm{~B}$ & $110.5(4)$ \\
\hline $\mathrm{C} 4 \mathrm{~A}-\mathrm{C} 5 \mathrm{~A}-\mathrm{H} 5 \mathrm{~A}$ & 121.3 & $\mathrm{C} 1 \mathrm{~B}-\mathrm{C} 7 \mathrm{~B}-\mathrm{S} 1 \mathrm{~B}$ & $120.4(4)$ \\
\hline $\mathrm{C} 6 \mathrm{~A}-\mathrm{C} 5 \mathrm{~A}-\mathrm{H} 5 \mathrm{~A}$ & 121.3 & $\mathrm{C} 7 \mathrm{~B}-\mathrm{C} 8 \mathrm{~B}-\mathrm{C} 9 \mathrm{~B}$ & $113.5(6)$ \\
\hline $\mathrm{C} 5 \mathrm{~A}-\mathrm{C} 6 \mathrm{~A}-\mathrm{C} 1 \mathrm{~A}$ & $122.4(5)$ & $\mathrm{C} 7 \mathrm{~B}-\mathrm{C} 8 \mathrm{~B}-\mathrm{H} 8 \mathrm{~B}$ & 123.3 \\
\hline $\mathrm{C} 5 \mathrm{~A}-\mathrm{C} 6 \mathrm{~A}-\mathrm{H} 6 \mathrm{~A}$ & 118.8 & $\mathrm{C} 9 \mathrm{~B}-\mathrm{C} 8 \mathrm{~B}-\mathrm{H} 8 \mathrm{~B}$ & 123.3 \\
\hline $\mathrm{C} 1 \mathrm{~A}-\mathrm{C} 6 \mathrm{~A}-\mathrm{H} 6 \mathrm{~A}$ & 118.8 & $\mathrm{C} 10 \mathrm{~B}-\mathrm{C} 9 \mathrm{~B}-\mathrm{C} 8 \mathrm{~B}$ & $113.4(5)$ \\
\hline $\mathrm{C} 8 \mathrm{~A}-\mathrm{C} 7 \mathrm{~A}-\mathrm{C} 1 \mathrm{~A}$ & $130.8(4)$ & $\mathrm{C} 10 \mathrm{~B}-\mathrm{C} 9 \mathrm{~B}-\mathrm{H} 9 \mathrm{~B}$ & 123.3 \\
\hline $\mathrm{C} 8 \mathrm{~A}-\mathrm{C} 7 \mathrm{~A}-\mathrm{S} 1 \mathrm{~A}$ & $109.9(4)$ & $\mathrm{C} 8 \mathrm{~B}-\mathrm{C} 9 \mathrm{~B}-\mathrm{H} 9 \mathrm{~B}$ & 123.3 \\
\hline $\mathrm{C} 1 \mathrm{~A}-\mathrm{C} 7 \mathrm{~A}-\mathrm{S} 1 \mathrm{~A}$ & $119.1(4)$ & $\mathrm{C} 9 \mathrm{~B}-\mathrm{C} 10 \mathrm{~B}-\mathrm{C} 11 \mathrm{~B}$ & $127.3(5)$ \\
\hline $\mathrm{C} 7 \mathrm{~A}-\mathrm{C} 8 \mathrm{~A}-\mathrm{C} 9 \mathrm{~A}$ & $113.5(5)$ & $\mathrm{C} 9 \mathrm{~B}-\mathrm{C} 10 \mathrm{~B}-\mathrm{S} 1 \mathrm{~B}$ & $110.0(4)$ \\
\hline $\mathrm{C} 7 \mathrm{~A}-\mathrm{C} 8 \mathrm{~A}-\mathrm{H} 8 \mathrm{~A}$ & 123.3 & $\mathrm{C} 11 \mathrm{~B}-\mathrm{C} 10 \mathrm{~B}-\mathrm{S} 1 \mathrm{~B}$ & $122.6(4)$ \\
\hline $\mathrm{C} 9 \mathrm{~A}-\mathrm{C} 8 \mathrm{~A}-\mathrm{H} 8 \mathrm{~A}$ & 123.3 & $\mathrm{C} 10 \mathrm{~B}-\mathrm{C} 11 \mathrm{~B}-\mathrm{C} 12 \mathrm{~B}$ & $111.7(4)$ \\
\hline $\mathrm{C} 10 \mathrm{~A}-\mathrm{C} 9 \mathrm{~A}-\mathrm{C} 8 \mathrm{~A}$ & $114.0(5)$ & $\mathrm{C} 10 \mathrm{~B}-\mathrm{C} 11 \mathrm{~B}-\mathrm{H} 11 \mathrm{C}$ & 109.3 \\
\hline $\mathrm{C} 10 \mathrm{~A}-\mathrm{C} 9 \mathrm{~A}-\mathrm{H} 9 \mathrm{~A}$ & 123.0 & $\mathrm{C} 12 \mathrm{~B}-\mathrm{C} 11 \mathrm{~B}-\mathrm{H} 11 \mathrm{C}$ & 109.3 \\
\hline $\mathrm{C} 8 \mathrm{~A}-\mathrm{C} 9 \mathrm{~A}-\mathrm{H} 9 \mathrm{~A}$ & 123.0 & $\mathrm{C} 10 \mathrm{~B}-\mathrm{C} 11 \mathrm{~B}-\mathrm{H} 11 \mathrm{D}$ & 109.3 \\
\hline
\end{tabular}




\begin{tabular}{|c|c|}
\hline $\mathrm{C} 9 \mathrm{~A}-\mathrm{C} 10 \mathrm{~A}-\mathrm{C} 11 \mathrm{~A}$ & $129.8(5)$ \\
\hline $\mathrm{C} 9 \mathrm{~A}-\mathrm{C} 10 \mathrm{~A}-\mathrm{S} 1 \mathrm{~A}$ & $109.6(4)$ \\
\hline $\mathrm{C} 11 \mathrm{~A}-\mathrm{C} 10 \mathrm{~A}-\mathrm{S} 1 \mathrm{~A}$ & $120.6(3)$ \\
\hline $\mathrm{C} 10 \mathrm{~A}-\mathrm{C} 11 \mathrm{~A}-\mathrm{C} 12 \mathrm{~A}$ & $115.7(4)$ \\
\hline $\mathrm{C} 10 \mathrm{~A}-\mathrm{C} 11 \mathrm{~A}-\mathrm{H} 11 \mathrm{~A}$ & 108.4 \\
\hline $\mathrm{C} 12 \mathrm{~A}-\mathrm{C} 11 \mathrm{~A}-\mathrm{H} 11 \mathrm{~A}$ & 108.4 \\
\hline $\mathrm{C} 10 \mathrm{~A}-\mathrm{C} 11 \mathrm{~A}-\mathrm{H} 11 \mathrm{~B}$ & 108.4 \\
\hline $\mathrm{C} 12 \mathrm{~A}-\mathrm{C} 11 \mathrm{~A}-\mathrm{H} 11 \mathrm{~B}$ & 108.4 \\
\hline $\mathrm{H} 11 \mathrm{~A}-\mathrm{C} 11 \mathrm{~A}-\mathrm{H} 11 \mathrm{~B}$ & 107.4 \\
\hline $\mathrm{C} 13 \mathrm{~A}-\mathrm{C} 12 \mathrm{~A}-\mathrm{C} 17 \mathrm{~A}$ & $119.1(4)$ \\
\hline $\mathrm{C} 13 \mathrm{~A}-\mathrm{C} 12 \mathrm{~A}-\mathrm{C} 11 \mathrm{~A}$ & $120.6(4)$ \\
\hline $\mathrm{C} 17 \mathrm{~A}-\mathrm{C} 12 \mathrm{~A}-\mathrm{C} 11 \mathrm{~A}$ & $120.3(4)$ \\
\hline $\mathrm{C} 14 \mathrm{~A}-\mathrm{C} 13 \mathrm{~A}-\mathrm{C} 12 \mathrm{~A}$ & $122.8(4)$ \\
\hline $\mathrm{C} 14 \mathrm{~A}-\mathrm{C} 13 \mathrm{~A}-\mathrm{H} 13 \mathrm{~A}$ & 118.6 \\
\hline $\mathrm{C} 12 \mathrm{~A}-\mathrm{C} 13 \mathrm{~A}-\mathrm{H} 13 \mathrm{~A}$ & 118.6 \\
\hline $\mathrm{C} 13 \mathrm{~A}-\mathrm{C} 14 \mathrm{~A}-\mathrm{C} 15 \mathrm{~A}$ & $117.8(4)$ \\
\hline $\mathrm{C} 13 \mathrm{~A}-\mathrm{C} 14 \mathrm{~A}-\mathrm{C} 19 \mathrm{~A}$ & $123.7(4)$ \\
\hline $\mathrm{C} 15 \mathrm{~A}-\mathrm{C} 14 \mathrm{~A}-\mathrm{C} 19 \mathrm{~A}$ & $118.5(4)$ \\
\hline $\mathrm{C} 16 \mathrm{~A}-\mathrm{C} 15 \mathrm{~A}-\mathrm{C} 14 \mathrm{~A}$ & $120.3(5)$ \\
\hline $\mathrm{C} 16 \mathrm{~A}-\mathrm{C} 15 \mathrm{~A}-\mathrm{H} 15 \mathrm{~A}$ & 119.8 \\
\hline $\mathrm{C} 14 \mathrm{~A}-\mathrm{C} 15 \mathrm{~A}-\mathrm{H} 15 \mathrm{~A}$ & 119.8 \\
\hline $\mathrm{C} 17 \mathrm{~A}-\mathrm{C} 16 \mathrm{~A}-\mathrm{C} 15 \mathrm{~A}$ & $121.7(5)$ \\
\hline $\mathrm{C} 17 \mathrm{~A}-\mathrm{C} 16 \mathrm{~A}-\mathrm{H} 16 \mathrm{~A}$ & 119.2 \\
\hline $\mathrm{C} 15 \mathrm{~A}-\mathrm{C} 16 \mathrm{~A}-\mathrm{H} 16 \mathrm{~A}$ & 119.2 \\
\hline $\mathrm{C} 16 \mathrm{~A}-\mathrm{C} 17 \mathrm{~A}-\mathrm{C} 12 \mathrm{~A}$ & $118.4(5)$ \\
\hline $\mathrm{C} 16 \mathrm{~A}-\mathrm{C} 17 \mathrm{~A}-\mathrm{C} 18 \mathrm{~A}$ & $120.0(4)$ \\
\hline $\mathrm{C} 12 \mathrm{~A}-\mathrm{C} 17 \mathrm{~A}-\mathrm{C} 18 \mathrm{~A}$ & $121.6(5)$ \\
\hline $\mathrm{C} 17 \mathrm{~A}-\mathrm{C} 18 \mathrm{~A}-\mathrm{H} 18 \mathrm{~A}$ & 109.5 \\
\hline $\mathrm{C} 17 \mathrm{~A}-\mathrm{C} 18 \mathrm{~A}-\mathrm{H} 18 \mathrm{~B}$ & 109.5 \\
\hline $\mathrm{H} 18 \mathrm{~A}-\mathrm{C} 18 \mathrm{~A}-\mathrm{H} 18 \mathrm{~B}$ & 109.5 \\
\hline $\mathrm{C} 17 \mathrm{~A}-\mathrm{C} 18 \mathrm{~A}-\mathrm{H} 18 \mathrm{C}$ & 109.5 \\
\hline $\mathrm{H} 18 \mathrm{~A}-\mathrm{C} 18 \mathrm{~A}-\mathrm{H} 18 \mathrm{C}$ & 109.5 \\
\hline $\mathrm{H} 18 \mathrm{~B}-\mathrm{C} 18 \mathrm{~A}-\mathrm{H} 18 \mathrm{C}$ & 109.5 \\
\hline $\mathrm{O} 1 \mathrm{~A}-\mathrm{C} 19 \mathrm{~A}-\mathrm{C} 14 \mathrm{~A}$ & $106.9(3)$ \\
\hline $\mathrm{O} 1 \mathrm{~A}-\mathrm{C} 19 \mathrm{~A}-\mathrm{C} 20 \mathrm{~A}$ & $109.0(3)$ \\
\hline $\mathrm{C} 14 \mathrm{~A}-\mathrm{C} 19 \mathrm{~A}-\mathrm{C} 20 \mathrm{~A}$ & $113.6(4)$ \\
\hline $\mathrm{O} 1 \mathrm{~A}-\mathrm{C} 19 \mathrm{~A}-\mathrm{H} 19 \mathrm{~A}$ & 109.1 \\
\hline $\mathrm{C} 14 \mathrm{~A}-\mathrm{C} 19 \mathrm{~A}-\mathrm{H} 19 \mathrm{~A}$ & 109.1 \\
\hline $\mathrm{C} 20 \mathrm{~A}-\mathrm{C} 19 \mathrm{~A}-\mathrm{H} 19 \mathrm{~A}$ & 109.1 \\
\hline $\mathrm{O} 2 \mathrm{~A}-\mathrm{C} 20 \mathrm{~A}-\mathrm{C} 21 \mathrm{~A}$ & $111.7(3)$ \\
\hline $\mathrm{O} 2 \mathrm{~A}-\mathrm{C} 20 \mathrm{~A}-\mathrm{C} 19 \mathrm{~A}$ & $108.2(3)$ \\
\hline $\mathrm{C} 21 \mathrm{~A}-\mathrm{C} 20 \mathrm{~A}-\mathrm{C} 19 \mathrm{~A}$ & $111.4(4)$ \\
\hline $\mathrm{O} 2 \mathrm{~A}-\mathrm{C} 20 \mathrm{~A}-\mathrm{H} 20 \mathrm{~A}$ & 108.5 \\
\hline $\mathrm{C} 21 \mathrm{~A}-\mathrm{C} 20 \mathrm{~A}-\mathrm{H} 20 \mathrm{~A}$ & 108.5 \\
\hline $\mathrm{C} 19 \mathrm{~A}-\mathrm{C} 20 \mathrm{~A}-\mathrm{H} 20 \mathrm{~A}$ & 108.5 \\
\hline $\mathrm{O} 3 \mathrm{~A}-\mathrm{C} 21 \mathrm{~A}-\mathrm{C} 22 \mathrm{~A}$ & $107.4(4)$ \\
\hline $\mathrm{O} 3 \mathrm{~A}-\mathrm{C} 21 \mathrm{~A}-\mathrm{C} 20 \mathrm{~A}$ & $111.7(4)$ \\
\hline $\mathrm{C} 22 \mathrm{~A}-\mathrm{C} 21 \mathrm{~A}-\mathrm{C} 20 \mathrm{~A}$ & $111.3(3)$ \\
\hline
\end{tabular}

\begin{tabular}{|c|c|}
\hline $\mathrm{C} 12 \mathrm{~B}-\mathrm{C} 11 \mathrm{~B}-\mathrm{H} 11 \mathrm{D}$ & 109.3 \\
\hline $\mathrm{H} 11 \mathrm{C}-\mathrm{C} 11 \mathrm{~B}-\mathrm{H} 11 \mathrm{D}$ & 107.9 \\
\hline $\mathrm{C} 13 \mathrm{~B}-\mathrm{C} 12 \mathrm{~B}-\mathrm{C} 17 \mathrm{~B}$ & $119.8(4)$ \\
\hline $\mathrm{C} 13 \mathrm{~B}-\mathrm{C} 12 \mathrm{~B}-\mathrm{C} 11 \mathrm{~B}$ & $117.5(5)$ \\
\hline $\mathrm{C} 17 \mathrm{~B}-\mathrm{C} 12 \mathrm{~B}-\mathrm{C} 11 \mathrm{~B}$ & $122.7(4)$ \\
\hline $\mathrm{C} 12 \mathrm{~B}-\mathrm{C} 13 \mathrm{~B}-\mathrm{C} 14 \mathrm{~B}$ & $121.4(4)$ \\
\hline $\mathrm{C} 12 \mathrm{~B}-\mathrm{C} 13 \mathrm{~B}-\mathrm{H} 13 \mathrm{~B}$ & 119.3 \\
\hline $\mathrm{C} 14 \mathrm{~B}-\mathrm{C} 13 \mathrm{~B}-\mathrm{H} 13 \mathrm{~B}$ & 119.3 \\
\hline $\mathrm{C} 15 \mathrm{~B}-\mathrm{C} 14 \mathrm{~B}-\mathrm{C} 13 \mathrm{~B}$ & $118.0(4)$ \\
\hline $\mathrm{C} 15 \mathrm{~B}-\mathrm{C} 14 \mathrm{~B}-\mathrm{C} 19 \mathrm{~B}$ & $121.6(4)$ \\
\hline $\mathrm{C} 13 \mathrm{~B}-\mathrm{C} 14 \mathrm{~B}-\mathrm{C} 19 \mathrm{~B}$ & $120.4(4)$ \\
\hline $\mathrm{C} 16 \mathrm{~B}-\mathrm{C} 15 \mathrm{~B}-\mathrm{C} 14 \mathrm{~B}$ & $120.4(4)$ \\
\hline $\mathrm{C} 16 \mathrm{~B}-\mathrm{C} 15 \mathrm{~B}-\mathrm{H} 15 \mathrm{~B}$ & 119.8 \\
\hline $\mathrm{C} 14 \mathrm{~B}-\mathrm{C} 15 \mathrm{~B}-\mathrm{H} 15 \mathrm{~B}$ & 119.8 \\
\hline $\mathrm{C} 15 \mathrm{~B}-\mathrm{C} 16 \mathrm{~B}-\mathrm{C} 17 \mathrm{~B}$ & $122.1(5)$ \\
\hline $\mathrm{C} 15 \mathrm{~B}-\mathrm{C} 16 \mathrm{~B}-\mathrm{H} 16 \mathrm{~B}$ & 118.9 \\
\hline $\mathrm{C} 17 \mathrm{~B}-\mathrm{C} 16 \mathrm{~B}-\mathrm{H} 16 \mathrm{~B}$ & 118.9 \\
\hline $\mathrm{C} 16 \mathrm{~B}-\mathrm{C} 17 \mathrm{~B}-\mathrm{C} 12 \mathrm{~B}$ & $118.3(4)$ \\
\hline $\mathrm{C} 16 \mathrm{~B}-\mathrm{C} 17 \mathrm{~B}-\mathrm{C} 18 \mathrm{~B}$ & $119.6(5)$ \\
\hline $\mathrm{C} 12 \mathrm{~B}-\mathrm{C} 17 \mathrm{~B}-\mathrm{C} 18 \mathrm{~B}$ & $122.0(5)$ \\
\hline $\mathrm{C} 17 \mathrm{~B}-\mathrm{C} 18 \mathrm{~B}-\mathrm{H} 18 \mathrm{D}$ & 109.5 \\
\hline $\mathrm{C} 17 \mathrm{~B}-\mathrm{C} 18 \mathrm{~B}-\mathrm{H} 18 \mathrm{E}$ & 109.5 \\
\hline $\mathrm{H} 18 \mathrm{D}-\mathrm{C} 18 \mathrm{~B}-\mathrm{H} 18 \mathrm{E}$ & 109.5 \\
\hline $\mathrm{C} 17 \mathrm{~B}-\mathrm{C} 18 \mathrm{~B}-\mathrm{H} 18 \mathrm{~F}$ & 109.5 \\
\hline $\mathrm{H} 18 \mathrm{D}-\mathrm{C} 18 \mathrm{~B}-\mathrm{H} 18 \mathrm{~F}$ & 109.5 \\
\hline $\mathrm{H} 18 \mathrm{E}-\mathrm{C} 18 \mathrm{~B}-\mathrm{H} 18 \mathrm{~F}$ & 109.5 \\
\hline $\mathrm{O} 1 \mathrm{~B}-\mathrm{C} 19 \mathrm{~B}-\mathrm{C} 14 \mathrm{~B}$ & $107.9(3)$ \\
\hline $\mathrm{O} 1 \mathrm{~B}-\mathrm{C} 19 \mathrm{~B}-\mathrm{C} 20 \mathrm{~B}$ & $109.9(3)$ \\
\hline $\mathrm{C} 14 \mathrm{~B}-\mathrm{C} 19 \mathrm{~B}-\mathrm{C} 20 \mathrm{~B}$ & $110.9(4)$ \\
\hline $\mathrm{O} 1 \mathrm{~B}-\mathrm{C} 19 \mathrm{~B}-\mathrm{H} 19 \mathrm{~B}$ & 109.4 \\
\hline $\mathrm{C} 14 \mathrm{~B}-\mathrm{C} 19 \mathrm{~B}-\mathrm{H} 19 \mathrm{~B}$ & 109.4 \\
\hline $\mathrm{C} 20 \mathrm{~B}-\mathrm{C} 19 \mathrm{~B}-\mathrm{H} 19 \mathrm{~B}$ & 109.4 \\
\hline $\mathrm{O} 2 \mathrm{~B}-\mathrm{C} 20 \mathrm{~B}-\mathrm{C} 21 \mathrm{~B}$ & $109.3(3)$ \\
\hline $\mathrm{O} 2 \mathrm{~B}-\mathrm{C} 20 \mathrm{~B}-\mathrm{C} 19 \mathrm{~B}$ & $107.4(3)$ \\
\hline $\mathrm{C} 21 \mathrm{~B}-\mathrm{C} 20 \mathrm{~B}-\mathrm{C} 19 \mathrm{~B}$ & $113.5(3)$ \\
\hline $\mathrm{O} 2 \mathrm{~B}-\mathrm{C} 20 \mathrm{~B}-\mathrm{H} 20 \mathrm{~B}$ & 108.8 \\
\hline $\mathrm{C} 21 \mathrm{~B}-\mathrm{C} 20 \mathrm{~B}-\mathrm{H} 20 \mathrm{~B}$ & 108.8 \\
\hline $\mathrm{C} 19 \mathrm{~B}-\mathrm{C} 20 \mathrm{~B}-\mathrm{H} 20 \mathrm{~B}$ & 108.8 \\
\hline $\mathrm{O} 3 \mathrm{~B}-\mathrm{C} 21 \mathrm{~B}-\mathrm{C} 22 \mathrm{~B}$ & $109.5(4)$ \\
\hline $\mathrm{O} 3 \mathrm{~B}-\mathrm{C} 21 \mathrm{~B}-\mathrm{C} 20 \mathrm{~B}$ & $109.8(4)$ \\
\hline $\mathrm{C} 22 \mathrm{~B}-\mathrm{C} 21 \mathrm{~B}-\mathrm{C} 20 \mathrm{~B}$ & $112.6(3)$ \\
\hline $\mathrm{O} 3 \mathrm{~B}-\mathrm{C} 21 \mathrm{~B}-\mathrm{H} 21 \mathrm{~B}$ & 108.3 \\
\hline $\mathrm{C} 22 \mathrm{~B}-\mathrm{C} 21 \mathrm{~B}-\mathrm{H} 21 \mathrm{~B}$ & 108.3 \\
\hline $\mathrm{C} 20 \mathrm{~B}-\mathrm{C} 21 \mathrm{~B}-\mathrm{H} 21 \mathrm{~B}$ & 108.3 \\
\hline $\mathrm{O} 4 \mathrm{~B}-\mathrm{C} 22 \mathrm{~B}-\mathrm{C} 21 \mathrm{~B}$ & $110.9(3)$ \\
\hline $\mathrm{O} 4 \mathrm{~B}-\mathrm{C} 22 \mathrm{~B}-\mathrm{C} 23 \mathrm{~B}$ & $106.5(3)$ \\
\hline $\mathrm{C} 21 \mathrm{~B}-\mathrm{C} 22 \mathrm{~B}-\mathrm{C} 23 \mathrm{~B}$ & $109.5(4)$ \\
\hline $\mathrm{O} 4 \mathrm{~B}-\mathrm{C} 22 \mathrm{~B}-\mathrm{H} 22 \mathrm{~B}$ & 109.9 \\
\hline
\end{tabular}




\begin{tabular}{|c|c|}
\hline $\mathrm{O} 3 \mathrm{~A}-\mathrm{C} 21 \mathrm{~A}-\mathrm{H} 21 \mathrm{~A}$ & 108.8 \\
\hline $\mathrm{C} 22 \mathrm{~A}-\mathrm{C} 21 \mathrm{~A}-\mathrm{H} 21 \mathrm{~A}$ & 108.8 \\
\hline $\mathrm{C} 20 \mathrm{~A}-\mathrm{C} 21 \mathrm{~A}-\mathrm{H} 21 \mathrm{~A}$ & 108.8 \\
\hline $\mathrm{O} 4 \mathrm{~A}-\mathrm{C} 22 \mathrm{~A}-\mathrm{C} 21 \mathrm{~A}$ & $106.9(3)$ \\
\hline $\mathrm{O} 4 \mathrm{~A}-\mathrm{C} 22 \mathrm{~A}-\mathrm{C} 23 \mathrm{~A}$ & $110.3(4)$ \\
\hline $\mathrm{C} 21 \mathrm{~A}-\mathrm{C} 22 \mathrm{~A}-\mathrm{C} 23 \mathrm{~A}$ & $111.2(4)$ \\
\hline $\mathrm{O} 4 \mathrm{~A}-\mathrm{C} 22 \mathrm{~A}-\mathrm{H} 22 \mathrm{~A}$ & 109.5 \\
\hline $\mathrm{C} 21 \mathrm{~A}-\mathrm{C} 22 \mathrm{~A}-\mathrm{H} 22 \mathrm{~A}$ & 109.5 \\
\hline $\mathrm{C} 23 \mathrm{~A}-\mathrm{C} 22 \mathrm{~A}-\mathrm{H} 22 \mathrm{~A}$ & 109.5 \\
\hline $\mathrm{O} 1 \mathrm{~A}-\mathrm{C} 23 \mathrm{~A}-\mathrm{C} 22 \mathrm{~A}$ & $109.8(4)$ \\
\hline $\mathrm{O} 1 \mathrm{~A}-\mathrm{C} 23 \mathrm{~A}-\mathrm{C} 24 \mathrm{~A}$ & $104.7(3)$ \\
\hline $\mathrm{C} 22 \mathrm{~A}-\mathrm{C} 23 \mathrm{~A}-\mathrm{C} 24 \mathrm{~A}$ & $108.2(4)$ \\
\hline $\mathrm{O} 1 \mathrm{~A}-\mathrm{C} 23 \mathrm{~A}-\mathrm{H} 23 \mathrm{~A}$ & 111.3 \\
\hline $\mathrm{C} 22 \mathrm{~A}-\mathrm{C} 23 \mathrm{~A}-\mathrm{H} 23 \mathrm{~A}$ & 111.3 \\
\hline $\mathrm{C} 24 \mathrm{~A}-\mathrm{C} 23 \mathrm{~A}-\mathrm{H} 23 \mathrm{~A}$ & 111.3 \\
\hline $\mathrm{O} 5 \mathrm{~A}-\mathrm{C} 24 \mathrm{~A}-\mathrm{C} 23 \mathrm{~A}$ & $108.1(5)$ \\
\hline $\mathrm{O} 5 \mathrm{~A}-\mathrm{C} 24 \mathrm{~A}-\mathrm{H} 24 \mathrm{~A}$ & 110.1 \\
\hline $\mathrm{C} 23 \mathrm{~A}-\mathrm{C} 24 \mathrm{~A}-\mathrm{H} 24 \mathrm{~A}$ & 110.1 \\
\hline $\mathrm{O} 5 \mathrm{~A}-\mathrm{C} 24 \mathrm{~A}-\mathrm{H} 24 \mathrm{~B}$ & 110.1 \\
\hline $\mathrm{C} 23 \mathrm{~A}-\mathrm{C} 24 \mathrm{~A}-\mathrm{H} 24 \mathrm{~B}$ & 110.1 \\
\hline $\mathrm{H} 24 \mathrm{~A}-\mathrm{C} 24 \mathrm{~A}-\mathrm{H} 24 \mathrm{~B}$ & 108.4 \\
\hline $\mathrm{C} 2 \mathrm{~B}-\mathrm{C} 1 \mathrm{~B}-\mathrm{C} 6 \mathrm{~B}$ & $117.1(6)$ \\
\hline $\mathrm{C} 2 \mathrm{~B}-\mathrm{C} 1 \mathrm{~B}-\mathrm{C} 7 \mathrm{~B}$ & $121.0(6)$ \\
\hline $\mathrm{C} 6 \mathrm{~B}-\mathrm{C} 1 \mathrm{~B}-\mathrm{C} 7 \mathrm{~B}$ & $121.8(5)$ \\
\hline $\mathrm{C} 3 \mathrm{~B}-\mathrm{C} 2 \mathrm{~B}-\mathrm{C} 1 \mathrm{~B}$ & $122.4(6)$ \\
\hline $\mathrm{C} 3 \mathrm{~B}-\mathrm{C} 2 \mathrm{~B}-\mathrm{H} 2 \mathrm{~B}$ & 118.8 \\
\hline $\mathrm{C} 1 \mathrm{~B}-\mathrm{C} 2 \mathrm{~B}-\mathrm{H} 2 \mathrm{~B}$ & 118.8 \\
\hline $\mathrm{C} 4 \mathrm{~B}-\mathrm{C} 3 \mathrm{~B}-\mathrm{C} 2 \mathrm{~B}$ & $117.5(6)$ \\
\hline $\mathrm{C} 6 \mathrm{~A}-\mathrm{C} 1 \mathrm{~A}-\mathrm{C} 2 \mathrm{~A}-\mathrm{C} 3 \mathrm{~A}$ & $-0.9(8)$ \\
\hline $\mathrm{C} 7 \mathrm{~A}-\mathrm{C} 1 \mathrm{~A}-\mathrm{C} 2 \mathrm{~A}-\mathrm{C} 3 \mathrm{~A}$ & $176.6(5)$ \\
\hline $\mathrm{C} 1 \mathrm{~A}-\mathrm{C} 2 \mathrm{~A}-\mathrm{C} 3 \mathrm{~A}-\mathrm{C} 4 \mathrm{~A}$ & $0.9(8)$ \\
\hline $\mathrm{C} 2 \mathrm{~A}-\mathrm{C} 3 \mathrm{~A}-\mathrm{C} 4 \mathrm{~A}-\mathrm{C} 5 \mathrm{~A}$ & $-1.0(10)$ \\
\hline $\mathrm{C} 2 \mathrm{~A}-\mathrm{C} 3 \mathrm{~A}-\mathrm{C} 4 \mathrm{~A}-\mathrm{F} 1 \mathrm{~A}$ & $-179.0(5)$ \\
\hline $\mathrm{C} 3 \mathrm{~A}-\mathrm{C} 4 \mathrm{~A}-\mathrm{C} 5 \mathrm{~A}-\mathrm{C} 6 \mathrm{~A}$ & $1.1(9)$ \\
\hline $\mathrm{F} 1 \mathrm{~A}-\mathrm{C} 4 \mathrm{~A}-\mathrm{C} 5 \mathrm{~A}-\mathrm{C} 6 \mathrm{~A}$ & $179.1(5)$ \\
\hline $\mathrm{C} 4 \mathrm{~A}-\mathrm{C} 5 \mathrm{~A}-\mathrm{C} 6 \mathrm{~A}-\mathrm{C} 1 \mathrm{~A}$ & $-1.0(9)$ \\
\hline $\mathrm{C} 2 \mathrm{~A}-\mathrm{C} 1 \mathrm{~A}-\mathrm{C} 6 \mathrm{~A}-\mathrm{C} 5 \mathrm{~A}$ & $0.9(8)$ \\
\hline $\mathrm{C} 7 \mathrm{~A}-\mathrm{C} 1 \mathrm{~A}-\mathrm{C} 6 \mathrm{~A}-\mathrm{C} 5 \mathrm{~A}$ & $-176.5(5)$ \\
\hline $\mathrm{C} 6 \mathrm{~A}-\mathrm{C} 1 \mathrm{~A}-\mathrm{C} 7 \mathrm{~A}-\mathrm{C} 8 \mathrm{~A}$ & $-161.5(6)$ \\
\hline $\mathrm{C} 2 \mathrm{~A}-\mathrm{C} 1 \mathrm{~A}-\mathrm{C} 7 \mathrm{~A}-\mathrm{C} 8 \mathrm{~A}$ & $21.2(8)$ \\
\hline $\mathrm{C} 6 \mathrm{~A}-\mathrm{C} 1 \mathrm{~A}-\mathrm{C} 7 \mathrm{~A}-\mathrm{S} 1 \mathrm{~A}$ & $24.2(6)$ \\
\hline $\mathrm{C} 2 \mathrm{~A}-\mathrm{C} 1 \mathrm{~A}-\mathrm{C} 7 \mathrm{~A}-\mathrm{S} 1 \mathrm{~A}$ & $-153.1(4)$ \\
\hline $\mathrm{C} 1 \mathrm{~A}-\mathrm{C} 7 \mathrm{~A}-\mathrm{C} 8 \mathrm{~A}-\mathrm{C} 9 \mathrm{~A}$ & $-174.5(5)$ \\
\hline $\mathrm{S} 1 \mathrm{~A}-\mathrm{C} 7 \mathrm{~A}-\mathrm{C} 8 \mathrm{~A}-\mathrm{C} 9 \mathrm{~A}$ & $0.2(6)$ \\
\hline $\mathrm{C} 7 \mathrm{~A}-\mathrm{C} 8 \mathrm{~A}-\mathrm{C} 9 \mathrm{~A}-\mathrm{C} 10 \mathrm{~A}$ & $0.9(7)$ \\
\hline $\mathrm{C} 8 \mathrm{~A}-\mathrm{C} 9 \mathrm{~A}-\mathrm{C} 10 \mathrm{~A}-\mathrm{C} 11 \mathrm{~A}$ & $178.1(5)$ \\
\hline $\mathrm{C} 8 \mathrm{~A}-\mathrm{C} 9 \mathrm{~A}-\mathrm{C} 10 \mathrm{~A}-\mathrm{S} 1 \mathrm{~A}$ & $-1.5(6)$ \\
\hline
\end{tabular}

$\begin{array}{ll}\mathrm{C} 21 \mathrm{~B}-\mathrm{C} 22 \mathrm{~B}-\mathrm{H} 22 \mathrm{~B} & 109.9 \\ \mathrm{C} 23 \mathrm{~B}-\mathrm{C} 22 \mathrm{~B}-\mathrm{H} 22 \mathrm{~B} & 109.9 \\ \mathrm{O} 1 \mathrm{~B}-\mathrm{C} 23 \mathrm{~B}-\mathrm{C} 24 \mathrm{~B} & 105.9(3) \\ \mathrm{O} 1 \mathrm{~B}-\mathrm{C} 23 \mathrm{~B}-\mathrm{C} 22 \mathrm{~B} & 107.6(3) \\ \mathrm{C} 24 \mathrm{~B}-\mathrm{C} 23 \mathrm{~B}-\mathrm{C} 22 \mathrm{~B} & 114.1(4) \\ \mathrm{O} 1 \mathrm{~B}-\mathrm{C} 23 \mathrm{~B}-\mathrm{H} 23 \mathrm{~B} & 109.7 \\ \mathrm{C} 24 \mathrm{~B}-\mathrm{C} 23 \mathrm{~B}-\mathrm{H} 23 \mathrm{~B} & 109.7 \\ \mathrm{C} 22 \mathrm{~B}-\mathrm{C} 23 \mathrm{~B}-\mathrm{H} 23 \mathrm{~B} & 109.7 \\ \text { O5B-C24B-C23B } & 108.5(4) \\ \mathrm{O} 5 \mathrm{~B}-\mathrm{C} 24 \mathrm{~B}-\mathrm{H} 24 \mathrm{C} & 110.0 \\ \mathrm{C} 23 \mathrm{~B}-\mathrm{C} 24 \mathrm{~B}-\mathrm{H} 24 \mathrm{C} & 110.0 \\ \mathrm{O} 5 \mathrm{~B}-\mathrm{C} 24 \mathrm{~B}-\mathrm{H} 24 \mathrm{D} & 110.0 \\ \mathrm{C} 23 \mathrm{~B}-\mathrm{C} 24 \mathrm{~B}-\mathrm{H} 24 \mathrm{D} & 110.0 \\ \mathrm{H} 24 \mathrm{C}-\mathrm{C} 24 \mathrm{~B}-\mathrm{H} 24 \mathrm{D} & 108.4 \\ \mathrm{C} 23 \mathrm{~A}-\mathrm{O} 1 \mathrm{~A}-\mathrm{C} 19 \mathrm{~A} & 114.3(3) \\ \mathrm{C} 20 \mathrm{~A}-\mathrm{O} 2 \mathrm{~A}-\mathrm{H} 2 \mathrm{~A} 1 & 109.5 \\ \mathrm{C} 21 \mathrm{~A}-\mathrm{O} 3 \mathrm{~A}-\mathrm{H} 3 \mathrm{~A} 1 & 109.5 \\ \mathrm{C} 22 \mathrm{~A}-\mathrm{O} 4 \mathrm{~A}-\mathrm{H} 4 \mathrm{~A} & 109.5 \\ \mathrm{C} 24 \mathrm{~A}-\mathrm{O} 5 \mathrm{~A}-\mathrm{H} 5 \mathrm{~A} 1 & 109.5 \\ \text { C19B-O1B-C23B } & 112.8(3) \\ \mathrm{C} 20 \mathrm{~B}-\mathrm{O} 2 \mathrm{~B}-\mathrm{H} 2 \mathrm{~B} 1 & 109.5 \\ \text { C21B-O3B-H3B1 } & 109.5 \\ \text { C22B-O4B-H4B } & 109.5 \\ \text { C24B-O5B-H5B1 } & 109.5 \\ \text { H61-O6-H62 } & 89.8 \\ \text { C10A-S1A-C7A } & 93.0(2) \\ \text { C10B-S1B-C7B } & 92.6(3) \\ & \end{array}$

$\begin{array}{ll}\mathrm{C} 7 \mathrm{~B}-\mathrm{C} 1 \mathrm{~B}-\mathrm{C} 6 \mathrm{~B}-\mathrm{C} 5 \mathrm{~B} & -176.1(5) \\ \mathrm{C} 4 \mathrm{~B}-\mathrm{C} 5 \mathrm{~B}-\mathrm{C} 6 \mathrm{~B}-\mathrm{C} 1 \mathrm{~B} & 1.6(10) \\ \mathrm{C} 2 \mathrm{~B}-\mathrm{C} 1 \mathrm{~B}-\mathrm{C} 7 \mathrm{~B}-\mathrm{C} 8 \mathrm{~B} & 20.5(9) \\ \mathrm{C} 6 \mathrm{~B}-\mathrm{C} 1 \mathrm{~B}-\mathrm{C} 7 \mathrm{~B}-\mathrm{C} 8 \mathrm{~B} & -162.9(6) \\ \mathrm{C} 2 \mathrm{~B}-\mathrm{C} 1 \mathrm{~B}-\mathrm{C} 7 \mathrm{~B}-\mathrm{S} 1 \mathrm{~B} & -156.6(4) \\ \mathrm{C} 6 \mathrm{~B}-\mathrm{C} 1 \mathrm{~B}-\mathrm{C} 7 \mathrm{~B}-\mathrm{S} 1 \mathrm{~B} & 20.1(7) \\ \mathrm{C} 1 \mathrm{~B}-\mathrm{C} 7 \mathrm{~B}-\mathrm{C} 8 \mathrm{~B}-\mathrm{C} 9 \mathrm{~B} & -177.8(5) \\ \mathrm{S} 1 \mathrm{~B}-\mathrm{C} 7 \mathrm{~B}-\mathrm{C} 8 \mathrm{~B}-\mathrm{C} 9 \mathrm{~B} & -0.5(6) \\ \mathrm{C} 7 \mathrm{~B}-\mathrm{C} 8 \mathrm{~B}-\mathrm{C} 9 \mathrm{~B}-\mathrm{C} 10 \mathrm{~B} & 0.6(7) \\ \mathrm{C} 8 \mathrm{~B}-\mathrm{C} 9 \mathrm{~B}-\mathrm{C} 10 \mathrm{~B}-\mathrm{C} 11 \mathrm{~B} & -178.3(5) \\ \mathrm{C} 8 \mathrm{~B}-\mathrm{C} 9 \mathrm{~B}-\mathrm{C} 10 \mathrm{~B}-\mathrm{S} 1 \mathrm{~B} & -0.3(6) \\ \mathrm{C} 9 \mathrm{~B}-\mathrm{C} 10 \mathrm{~B}-\mathrm{C} 11 \mathrm{~B}-\mathrm{C} 12 \mathrm{~B} & 108.0(6) \\ \mathrm{S} 1 \mathrm{~B}-\mathrm{C} 10 \mathrm{~B}-\mathrm{C} 11 \mathrm{~B}-\mathrm{C} 12 \mathrm{~B} & -69.8(5) \\ \mathrm{C} 10 \mathrm{~B}-\mathrm{C} 11 \mathrm{~B}-\mathrm{C} 12 \mathrm{~B}-\mathrm{C} 13 \mathrm{~B} & -84.3(5) \\ \mathrm{C} 10 \mathrm{~B}-\mathrm{C} 11 \mathrm{~B}-\mathrm{C} 12 \mathrm{~B}-\mathrm{C} 17 \mathrm{~B} & 93.7(6) \\ \mathrm{C} 17 \mathrm{~B}-\mathrm{C} 12 \mathrm{~B}-\mathrm{C} 13 \mathrm{~B}-\mathrm{C} 14 \mathrm{~B} & -0.6(7) \\ \mathrm{C} 11 \mathrm{~B}-\mathrm{C} 12 \mathrm{~B}-\mathrm{C} 13 \mathrm{~B}-\mathrm{C} 14 \mathrm{~B} & 177.4(4) \\ \mathrm{C} 12 \mathrm{~B}-\mathrm{C} 13 \mathrm{~B}-\mathrm{C} 14 \mathrm{~B}-\mathrm{C} 15 \mathrm{~B} & -0.7(6) \\ \mathrm{C} 12 \mathrm{~B}-\mathrm{C} 13 \mathrm{~B}-\mathrm{C} 14 \mathrm{~B}-\mathrm{C} 19 \mathrm{~B} & 177.0(4)\end{array}$




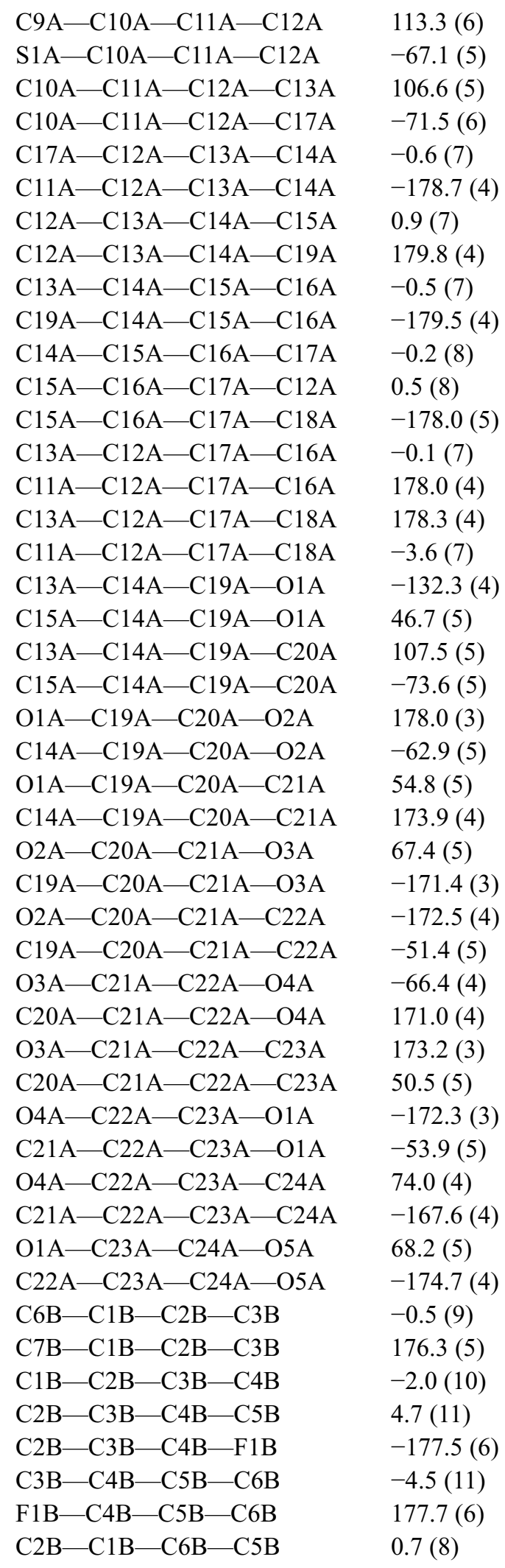

$113.3(6)$

$-67.1(5)$

$106.6(5)$

$-71.5(6)$

$-0.6(7)$

$-178.7(4)$

$0.9(7)$

$179.8(4)$

$-0.5(7)$

$-179.5(4)$

$-0.2(8)$

$0.5(8)$

$-178.0(5)$

$-0.1(7)$

$178.0(4)$

178.3 (4)

$-3.6(7)$

-132.3 (4)

46.7 (5)

107.5 (5)

$-73.6(5)$

178.0 (3)

$-62.9(5)$

$54.8(5)$

173.9 (4)

67.4 (5)

$-171.4(3)$

-172.5 (4)

$-51.4(5)$

-66.4 (4)

171.0 (4)

$173.2(3)$

50.5 (5)

$-172.3(3)$

$-53.9(5)$

74.0 (4)

-167.6 (4)

$68.2(5)$

-174.7 (4)

-0.5 (9)

$176.3(5)$

$-2.0(10)$

4.7 (11)

$-177.5(6)$

$-4.5(11)$

177.7 (6)

$0.7(8)$

\begin{tabular}{|c|c|}
\hline $\mathrm{C} 13 \mathrm{~B}-\mathrm{C} 14 \mathrm{~B}-\mathrm{C} 15 \mathrm{~B}-\mathrm{C} 16 \mathrm{~B}$ & $1.4(6)$ \\
\hline $\mathrm{C} 19 \mathrm{~B}-\mathrm{C} 14 \mathrm{~B}-\mathrm{C} 15 \mathrm{~B}-\mathrm{C} 16 \mathrm{~B}$ & $-176.2(4)$ \\
\hline $\mathrm{C} 14 \mathrm{~B}-\mathrm{C} 15 \mathrm{~B}-\mathrm{C} 16 \mathrm{~B}-\mathrm{C} 17 \mathrm{~B}$ & $-0.9(7)$ \\
\hline $\mathrm{C} 15 \mathrm{~B}-\mathrm{C} 16 \mathrm{~B}-\mathrm{C} 17 \mathrm{~B}-\mathrm{C} 12 \mathrm{~B}$ & $-0.4(7)$ \\
\hline $\mathrm{C} 15 \mathrm{~B}-\mathrm{C} 16 \mathrm{~B}-\mathrm{C} 17 \mathrm{~B}-\mathrm{C} 18 \mathrm{~B}$ & $178.1(4)$ \\
\hline $\mathrm{C} 13 \mathrm{~B}-\mathrm{C} 12 \mathrm{~B}-\mathrm{C} 17 \mathrm{~B}-\mathrm{C} 16 \mathrm{~B}$ & $1.1(7)$ \\
\hline $\mathrm{C} 11 \mathrm{~B}-\mathrm{C} 12 \mathrm{~B}-\mathrm{C} 17 \mathrm{~B}-\mathrm{C} 16 \mathrm{~B}$ & $-176.8(4)$ \\
\hline $\mathrm{C} 13 \mathrm{~B}-\mathrm{C} 12 \mathrm{~B}-\mathrm{C} 17 \mathrm{~B}-\mathrm{C} 18 \mathrm{~B}$ & $-177.3(4)$ \\
\hline $\mathrm{C} 11 \mathrm{~B}-\mathrm{C} 12 \mathrm{~B}-\mathrm{C} 17 \mathrm{~B}-\mathrm{C} 18 \mathrm{~B}$ & $4.7(7)$ \\
\hline $\mathrm{C} 15 \mathrm{~B}-\mathrm{C} 14 \mathrm{~B}-\mathrm{C} 19 \mathrm{~B}-\mathrm{O} 1 \mathrm{~B}$ & $-141.6(4)$ \\
\hline $\mathrm{C} 13 \mathrm{~B}-\mathrm{C} 14 \mathrm{~B}-\mathrm{C} 19 \mathrm{~B}-\mathrm{O} 1 \mathrm{~B}$ & $40.9(5)$ \\
\hline $\mathrm{C} 15 \mathrm{~B}-\mathrm{C} 14 \mathrm{~B}-\mathrm{C} 19 \mathrm{~B}-\mathrm{C} 20 \mathrm{~B}$ & $98.1(5)$ \\
\hline $\mathrm{C} 13 \mathrm{~B}-\mathrm{C} 14 \mathrm{~B}-\mathrm{C} 19 \mathrm{~B}-\mathrm{C} 20 \mathrm{~B}$ & $-79.5(5)$ \\
\hline $\mathrm{O} 1 \mathrm{~B}-\mathrm{C} 19 \mathrm{~B}-\mathrm{C} 20 \mathrm{~B}-\mathrm{O} 2 \mathrm{~B}$ & $167.9(3)$ \\
\hline $\mathrm{C} 14 \mathrm{~B}-\mathrm{C} 19 \mathrm{~B}-\mathrm{C} 20 \mathrm{~B}-\mathrm{O} 2 \mathrm{~B}$ & $-72.9(4)$ \\
\hline $\mathrm{O} 1 \mathrm{~B}-\mathrm{C} 19 \mathrm{~B}-\mathrm{C} 20 \mathrm{~B}-\mathrm{C} 21 \mathrm{~B}$ & $46.9(5)$ \\
\hline $\mathrm{C} 14 \mathrm{~B}-\mathrm{C} 19 \mathrm{~B}-\mathrm{C} 20 \mathrm{~B}-\mathrm{C} 21 \mathrm{~B}$ & $166.2(4)$ \\
\hline $\mathrm{O} 2 \mathrm{~B}-\mathrm{C} 20 \mathrm{~B}-\mathrm{C} 21 \mathrm{~B}-\mathrm{O} 3 \mathrm{~B}$ & $73.6(4)$ \\
\hline $\mathrm{C} 19 \mathrm{~B}-\mathrm{C} 20 \mathrm{~B}-\mathrm{C} 21 \mathrm{~B}-\mathrm{O} 3 \mathrm{~B}$ & $-166.5(3)$ \\
\hline $\mathrm{O} 2 \mathrm{~B}-\mathrm{C} 20 \mathrm{~B}-\mathrm{C} 21 \mathrm{~B}-\mathrm{C} 22 \mathrm{~B}$ & $-164.1(3)$ \\
\hline $\mathrm{C} 19 \mathrm{~B}-\mathrm{C} 20 \mathrm{~B}-\mathrm{C} 21 \mathrm{~B}-\mathrm{C} 22 \mathrm{~B}$ & $-44.2(5)$ \\
\hline $\mathrm{O} 3 \mathrm{~B}-\mathrm{C} 21 \mathrm{~B}-\mathrm{C} 22 \mathrm{~B}-\mathrm{O} 4 \mathrm{~B}$ & $-69.7(4)$ \\
\hline $\mathrm{C} 20 \mathrm{~B}-\mathrm{C} 21 \mathrm{~B}-\mathrm{C} 22 \mathrm{~B}-\mathrm{O} 4 \mathrm{~B}$ & $167.8(3)$ \\
\hline $\mathrm{O} 3 \mathrm{~B}-\mathrm{C} 21 \mathrm{~B}-\mathrm{C} 22 \mathrm{~B}-\mathrm{C} 23 \mathrm{~B}$ & $173.0(3)$ \\
\hline $\mathrm{C} 20 \mathrm{~B}-\mathrm{C} 21 \mathrm{~B}-\mathrm{C} 22 \mathrm{~B}-\mathrm{C} 23 \mathrm{~B}$ & $50.5(5)$ \\
\hline $\mathrm{O} 4 \mathrm{~B}-\mathrm{C} 22 \mathrm{~B}-\mathrm{C} 23 \mathrm{~B}-\mathrm{O} 1 \mathrm{~B}$ & $179.2(3)$ \\
\hline $\mathrm{C} 21 \mathrm{~B}-\mathrm{C} 22 \mathrm{~B}-\mathrm{C} 23 \mathrm{~B}-\mathrm{O} 1 \mathrm{~B}$ & $-60.8(4)$ \\
\hline $\mathrm{O} 4 \mathrm{~B}-\mathrm{C} 22 \mathrm{~B}-\mathrm{C} 23 \mathrm{~B}-\mathrm{C} 24 \mathrm{~B}$ & $62.0(5)$ \\
\hline $\mathrm{C} 21 \mathrm{~B}-\mathrm{C} 22 \mathrm{~B}-\mathrm{C} 23 \mathrm{~B}-\mathrm{C} 24 \mathrm{~B}$ & $-178.0(4)$ \\
\hline $\mathrm{O} 1 \mathrm{~B}-\mathrm{C} 23 \mathrm{~B}-\mathrm{C} 24 \mathrm{~B}-\mathrm{O} 5 \mathrm{~B}$ & $67.8(4)$ \\
\hline $\mathrm{C} 22 \mathrm{~B}-\mathrm{C} 23 \mathrm{~B}-\mathrm{C} 24 \mathrm{~B}-\mathrm{O} 5 \mathrm{~B}$ & $-174.0(4)$ \\
\hline $\mathrm{C} 22 \mathrm{~A}-\mathrm{C} 23 \mathrm{~A}-\mathrm{O} 1 \mathrm{~A}-\mathrm{C} 19 \mathrm{~A}$ & $61.0(5)$ \\
\hline $\mathrm{C} 24 \mathrm{~A}-\mathrm{C} 23 \mathrm{~A}-\mathrm{O} 1 \mathrm{~A}-\mathrm{C} 19 \mathrm{~A}$ & $176.9(4)$ \\
\hline $\mathrm{C} 14 \mathrm{~A}-\mathrm{C} 19 \mathrm{~A}-\mathrm{O} 1 \mathrm{~A}-\mathrm{C} 23 \mathrm{~A}$ & $175.6(4)$ \\
\hline $\mathrm{C} 20 \mathrm{~A}-\mathrm{C} 19 \mathrm{~A}-\mathrm{O} 1 \mathrm{~A}-\mathrm{C} 23 \mathrm{~A}$ & $-61.2(5)$ \\
\hline $\mathrm{C} 14 \mathrm{~B}-\mathrm{C} 19 \mathrm{~B}-\mathrm{O} 1 \mathrm{~B}-\mathrm{C} 23 \mathrm{~B}$ & $178.8(3)$ \\
\hline $\mathrm{C} 20 \mathrm{~B}-\mathrm{C} 19 \mathrm{~B}-\mathrm{O} 1 \mathrm{~B}-\mathrm{C} 23 \mathrm{~B}$ & $-60.1(4)$ \\
\hline $\mathrm{C} 24 \mathrm{~B}-\mathrm{C} 23 \mathrm{~B}-\mathrm{O} 1 \mathrm{~B}-\mathrm{C} 19 \mathrm{~B}$ & $-169.8(3)$ \\
\hline $\mathrm{C} 22 \mathrm{~B}-\mathrm{C} 23 \mathrm{~B}-\mathrm{O} 1 \mathrm{~B}-\mathrm{C} 19 \mathrm{~B}$ & $67.7(4)$ \\
\hline $\mathrm{C} 9 \mathrm{~A}-\mathrm{C} 10 \mathrm{~A}-\mathrm{S} 1 \mathrm{~A}-\mathrm{C} 7 \mathrm{~A}$ & $1.4(4)$ \\
\hline $\mathrm{C} 11 \mathrm{~A}-\mathrm{C} 10 \mathrm{~A}-\mathrm{S} 1 \mathrm{~A}-\mathrm{C} 7 \mathrm{~A}$ & $-178.3(4)$ \\
\hline $\mathrm{C} 8 \mathrm{~A}-\mathrm{C} 7 \mathrm{~A}-\mathrm{S} 1 \mathrm{~A}-\mathrm{C} 10 \mathrm{~A}$ & $-0.9(4)$ \\
\hline $\mathrm{C} 1 \mathrm{~A}-\mathrm{C} 7 \mathrm{~A}-\mathrm{S} 1 \mathrm{~A}-\mathrm{C} 10 \mathrm{~A}$ & $174.5(4)$ \\
\hline $\mathrm{C} 9 \mathrm{~B}-\mathrm{C} 10 \mathrm{~B}-\mathrm{S} 1 \mathrm{~B}-\mathrm{C} 7 \mathrm{~B}$ & $0.0(4)$ \\
\hline $\mathrm{C} 11 \mathrm{~B}-\mathrm{C} 10 \mathrm{~B}-\mathrm{S} 1 \mathrm{~B}-\mathrm{C} 7 \mathrm{~B}$ & $178.1(4)$ \\
\hline $\mathrm{C} 8 \mathrm{~B}-\mathrm{C} 7 \mathrm{~B}-\mathrm{S} 1 \mathrm{~B}-\mathrm{C} 10 \mathrm{~B}$ & $0.3(4)$ \\
\hline $\mathrm{C} 1 \mathrm{~B}-\mathrm{C} 7 \mathrm{~B}-\mathrm{S} 1 \mathrm{~B}-\mathrm{C} 10 \mathrm{~B}$ & $177.8(4)$ \\
\hline
\end{tabular}


supporting information

Hydrogen-bond geometry $\left(\AA,{ }^{\circ}\right)$

\begin{tabular}{lllll}
\hline$D-\mathrm{H} \cdots A$ & $D-\mathrm{H}$ & $\mathrm{H} \cdots A$ & $D \cdots A$ & $D-\mathrm{H} \cdots A$ \\
\hline $\mathrm{O} 2 A-\mathrm{H} 2 A 1 \cdots \mathrm{O} 2 B^{\mathrm{i}}$ & 0.82 & 2.42 & $2.841(4)$ & 113 \\
$\mathrm{O} 3 A-\mathrm{H} 3 A 1 \cdots \mathrm{O} 2 B^{\mathrm{i}}$ & 0.82 & 2.17 & $2.951(4)$ & 158 \\
$\mathrm{O} 4 A-\mathrm{H} 4 A \cdots \mathrm{O} 5 B^{\mathrm{ii}}$ & 0.82 & 1.98 & $2.756(5)$ & 157 \\
$\mathrm{O} 2 B-\mathrm{H} 2 B 1 \cdots \mathrm{O} 4 A^{\mathrm{iii}}$ & 0.82 & 1.85 & $2.672(4)$ & 179 \\
$\mathrm{O} 3 B-\mathrm{H} 3 B \cdots \mathrm{O} 4 B^{\mathrm{i}}$ & 0.82 & 1.99 & $2.797(4)$ & 168 \\
$\mathrm{O} 4 B-\mathrm{H} 4 B \cdots \mathrm{O} 6$ & 0.82 & 1.93 & $2.749(5)$ & 172 \\
$\mathrm{O} 5 B-\mathrm{H} 5 B \cdots \mathrm{O} 3 B^{\mathrm{iv}}$ & 0.82 & 2.31 & $3.015(5)$ & 144 \\
$\mathrm{O} 6-\mathrm{H} 61 \cdots \mathrm{O} 2 A$ & 0.82 & 2.23 & $3.031(5)$ & 166 \\
$\mathrm{O} 6-\mathrm{H} 62 \cdots \mathrm{O} 3 A^{\mathrm{v}}$ & 0.83 & 2.30 & $3.058(5)$ & 153
\end{tabular}

Symmetry codes: (i) $x-1 / 2,-y+3 / 2,-z+1$; (ii) $x-1, y-1, z$; (iii) $x, y+1, z$; (iv) $x+1, y, z$; (v) $x+1 / 2,-y+1 / 2,-z+1$. 